\title{
HIGH-RESOLUTION CMB POWER SPECTRUM FROM THE COMPLETE ACBAR DATA SET
}

\author{
C. L. ReichardT ${ }^{1}$, P. A. R. AdE ${ }^{2}$, J. J. Bock ${ }^{1,3}$, J. R. Bond ${ }^{4}$, J. A. BreviK ${ }^{1}$, C. R. Contaldi ${ }^{5}$, M. D. Daub ${ }^{6}$,

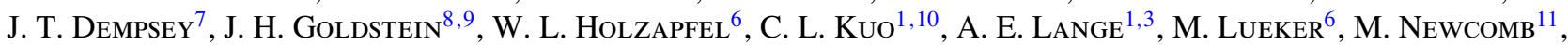 \\ J. B. Peterson ${ }^{12}$, J. Ruhl ${ }^{8}$, M. C. RunYan ${ }^{1}$, And Z. Staniszewski ${ }^{8}$

\begin{abstract}
In this paper, we present results from the complete set of cosmic microwave background (CMB) radiation temperature anisotropy observations made with the Arcminute Cosmology Bolometer Array Receiver (ACBAR) operating at $150 \mathrm{GHz}$. We include new data from the final 2005 observing season, expanding the number of detector hours by $210 \%$ and the sky coverage by $490 \%$ over that used for the previous ACBAR release. As a result, the band-power uncertainties have been reduced by more than a factor of two on angular scales encompassing the third to fifth acoustic peaks as well as the damping tail of the CMB power spectrum. The calibration uncertainty has been reduced from $6 \%$ to $2.1 \%$ in temperature through a direct comparison of the CMB anisotropy measured by ACBAR with that of the dipole-calibrated WMAP5 experiment. The measured power spectrum is consistent with a spatially flat, $\Lambda \mathrm{CDM}$ cosmological model. We include the effects of weak lensing in the power spectrum model computations and find that this significantly improves the fits of the models to the combined ACBAR+WMAP5 power spectrum. The preferred strength of the lensing is consistent with theoretical expectations. On fine angular scales, there is weak evidence $(1.1 \sigma)$ for excess power above the level expected from primary anisotropies. We expect any excess power to be dominated by the combination of emission from dusty protogalaxies and the Sunyaev-Zel'dovich effect (SZE). However, the excess observed by ACBAR is significantly smaller than the excess power at $\ell>2000$ reported by the CBI experiment operating at $30 \mathrm{GHz}$. Therefore, while it is unlikely that the CBI excess has a primordial origin; the combined ACBAR and $\mathrm{CBI}$ results are consistent with the source of the CBI excess being either the SZE or radio source contamination.
\end{abstract}

Key words: cosmic microwave background - cosmology: observations

\section{INTRODUCTION}

Observations of the cosmic microwave background (CMB) are among the most powerful and important tests of cosmological theory. Measurements of the angular power spectrum of CMB temperature anisotropies on angular scales $>10^{\prime}-$ corresponding to multipoles $\ell \lesssim 1000$ (Spergel et al. 2006) - in conjunction with other cosmological probes (Burles et al. 2001; Cole et al. 2005; Tegmark et al. 2006; Riess et al. 2007) have produced compelling evidence for the $\Lambda \mathrm{CDM}$ cosmological model. At higher multipoles, measurements probe the Silk damping tail of the power spectrum and provide an independent check of the cosmological model.

At smaller angular scales, the primary CMB anisotropies originating at redshift $z=1100$ are exponentially damped by photon diffusion. This effect, known as Silk damping, makes secondary anisotropies - those induced along the line of sight at lower redshift-increasingly important at higher $\ell$. At $150 \mathrm{GHz}$, for example, the Sunyaev-Zel'dovich effect (SZE) is expected to be brighter than the primary CMB anisotropy at $\ell \gtrsim 2500$. The amplitude of the SZE depends sensitively on the amplitude of the matter perturbations, scaling as $\sigma_{8}^{7}$. Measurements of the
CMB power spectrum with sufficient sensitivity on arcminute scales not only extend tests of the $\Lambda \mathrm{CDM}$ model's ability to accurately predict the features in the power spectrum of primary CMB anisotropy, but also probe the epoch of cluster formation and provide an independent measure of $\sigma_{8}$.

In this paper, we present the complete results of observations of CMB temperature anisotropies at $150 \mathrm{GHz}$ with $5^{\prime}$ resolution from the Arcminute Cosmology Bolometer Array Receiver (ACBAR) experiment at the South Pole station. Previous measurements of the CMB power spectrum by ACBAR have been presented in (Kuo et al. 2004, hereafter K04) and (Kuo et al. 2007, hereafter K07). In addition, the angular power spectrum on these angular scales has been measured at $30 \mathrm{GHz}$ by CBI (Readhead et al. 2004a), VSA (Dickinson et al. 2004), and BIMA (Dawson et al. 2006), and at 100 and $150 \mathrm{GHz}$ by QuAD (Ade et al. 2007).

To date, measurements at angular scales $<10^{\prime}$ have been consistent with predictions of the primary anisotropy based on measurements at larger angular scales with one exception. Both CBI (Mason et al. 2003; Bond et al. 2005) and BIMA (Dawson et al. 2006) observe excess power for $\ell>2000$ at $30 \mathrm{GHz}$ compared to the predictions of the $\Lambda \mathrm{CDM}$ model. This excess 
Table 1

CMB Fields

\begin{tabular}{lcccccc}
\hline \hline Field & R.A. $(\mathrm{deg})$ & Decl. $(\mathrm{deg})$ & Area $\left(\mathrm{deg}^{2}\right)$ & Year & \# of Detectors & Detector Hours \\
\hline CMB2(CMB4) & 73.963 & -46.268 & $26(17)$ & $2001(2002)$ & $4(8)$ & $2.0 \mathrm{k}(1.1 \mathrm{k})$ \\
CMB5 & 43.371 & -54.836 & 28 & $2002(2005)$ & $8(16)$ & $13.2 \mathrm{k}(10.6 \mathrm{k})$ \\
CMB6 & 32.693 & -50.983 & 23 & 2002 & 8 & $2.8 \mathrm{k}$ \\
CMB7(ext*) & 338.805 & -48.600 & $28(107)$ & $2002(2005)$ & $8(16)$ & $3.4 \mathrm{k}(12.4 \mathrm{k})$ \\
CMB8 & 82.297 & -46.598 & 61 & 2005 & 16 & $17.1 \mathrm{k}$ \\
CMB9* & 359.818 & -53.135 & 93 & 2005 & 16 & $4.0 \mathrm{k}$ \\
CMB10* & 19.544 & -53.171 & 93 & 2005 & 16 & $3.6 \mathrm{k}$ \\
CMB11* & 339.910 & -64.178 & 91 & 2005 & 16 & $4.9 \mathrm{k}$ \\
CMB12* & 21.849 & -64.197 & 92 & 2005 & 16 & $2.7 \mathrm{k}$ \\
CMB13* & 43.732 & -59.871 & 78 & 2005 & 16 & $7.6 \mathrm{k}$ \\
\hline
\end{tabular}

Notes. Central coordinates and size of each CMB field observed by ACBAR. The sixth column gives the number of $150 \mathrm{GHz}$ detectors. The last column gives the detector integration time for each field after cuts. The detector sensitivity was comparable (within $\sim 10 \%$ ) between 2002 and 2005. The six largest fields (marked with an asterisk*) are used in the calibration to WMAP. Note that the 2005 observations extended the declination range of the CMB7 field, leading to the combined field CMB7(ext). CMB2(CMB4) and CMB8 also partially overlap, but are analyzed separately for computational reasons. Approximately $1 / 4$ of the CMB2(CMB4) scans have been discarded to eliminate the overlapping coverage. The listed numbers reflect this loss.

can be explained by the SZE if $\sigma_{8} \approx 1$, but this value is in tension with the best-fit WMAP5 value of $\sigma_{8} \approx 0.8$. In K07, we found that while the frequency dependence of the excess is consistent with the SZE, the ACBAR and CBI data could not be used to rule out radio source contamination or systematic errors as the source of the CBI excess. Careful measurements over a broad range of frequencies and angular scales are needed to provide a definitive answer.

Current estimates of the primordial power spectrum are consistent with the predictions of slow-roll inflation for a nearly scale-invariant spectrum which may also include a small running of the spectral index. Sparked by the modest evidence for negative running in the WMAP first-year data, a number of authors have investigated how existing data sets limit the allowed inflationary scenarios (Peiris et al. 2003; Mukherjee \& Wang 2003; Bridle et al. 2003; Leach \& Liddle 2003). Small-scale data extend the range over which the primordial power spectrum is measured and can potentially yield information about the mechanism of inflation.

This is the third and final ACBAR power spectrum release. The first release in K04 analyzed two fields from the 2001 and 2002 seasons with a conservative field differencing algorithm. The second ACBAR power spectrum, presented by K07, added two more fields from the 2002 season and implemented an improved, undifferenced lead-main-trail (no LMT) analysis of the data set. The results presented here improve on the previous work in two ways. First, we include seven additional fields observed in the 2005 austral winter. These fields double the total number of detector hours and substantially improve the precision of the band-power estimates. In particular, the new fields were selected to dramatically expand ACBAR's sky coverage in order to reduce the cosmic variance contribution to the uncertainty and to improve the multipole resolution on angular scales below $\ell \lesssim 1800$. This angular range covers the third to fifth acoustic peaks, making it especially interesting for constraining cosmological models. Second, we implement a new temperature calibration based on a comparison of $\mathrm{CMB}$ fluctuations as measured by ACBAR and the WMAP satellite (Hinshaw et al. 2008). This improved calibration tightens constraints on cosmological models found from the combination of high- $\ell$ ACBAR band powers with low- $\ell$ results from other experiments.

This paper is organized as follows. In Section 2, we review the ACBAR instrument and the CMB observation program. The analysis algorithm is explained in Section 3. Section 4 is an overview of the calibration; the details of cross-calibration between WMAP5 and ACBAR are discussed in the Appendices. Information on ACBAR's beams can be found in Section 5. Systematic tests and foreground contamination are discussed in Section 6. We present the band-power results in Section 7, including a discussion of the scientific interpretation. The ACBAR band powers are combined with the results of other experiments to place constraints on the parameters of cosmological models in Section 8. In Section 9, we summarize the main results of this paper.

\section{THE INSTRUMENT AND OBSERVATIONS}

The ACBAR receiver was designed to take advantage of the excellent observing conditions at the South Pole to make extremely deep maps of CMB anisotropies (Runyan et al. 2003). It observes from the Viper telescope, a $2.1 \mathrm{~m}$ off-axis Gregorian with a beam size of $5^{\prime}$ at $150 \mathrm{GHz}$. The beams are swept across the sky at near-constant elevation by the motion of an actuated flat tertiary mirror.

The receiver contains 16 optically active bolometers cooled to $240 \mathrm{mK}$ by a three-stage $\mathrm{He}^{3}-\mathrm{He}^{3}-\mathrm{He}^{4}$ sorption refrigerator. The results reported here are derived from the $150 \mathrm{GHz}$ detectors: there were 4-150 GHz bolometers in 2001, 8 in 2002 and 2004, and 16 in 2005. The detectors were background limited at $150 \mathrm{GHz}$ with a sensitivity of approximately $340 \mu \mathrm{K} \sqrt{s}$.

In total, ACBAR observed 10 independent CMB fields detailed in Table 1 . The power spectrum derived from portions of four fields, CMB2/4, CMB5, CMB6, and CMB7 was reported in K07. Since then, we have completed the analysis of six new fields observed in 2005 as well as additional observations of the original four fields. Details of the instrument configuration and performance in the 2001 and 2002 seasons are given in Runyan et al. (2003), while additional details of the CMB observations, 
data reduction procedures, and beam maps can be found in $\mathrm{K} 04$ and $\mathrm{K} 07$.

\section{UNDIFFERENCED POWER SPECTRUM ANALYSIS}

Following the conventions of the previous data releases, the band powers $\mathbf{q}$ are reported in units of $\mu K^{2}$, and are used to parameterize the power spectrum according to

$$
\ell(\ell+1) C_{\ell} / 2 \pi \equiv \mathcal{D}_{\ell}=\sum_{B} q_{B} \chi_{B \ell},
$$

where $\chi_{B \ell}$ are top-hat functions; $\chi_{B \ell}=1$ for $\ell \in B$, and $\chi_{B \ell}=0$ for $\ell \notin B$. The ACBAR observations were carried out in an LMT pattern. Originally, the three fields were differenced according to the formula $M-(L+T) / 2$ in order to remove timedependent chopper synchronous offsets. In K07, this conservative strategy was shown to be unnecessary and an undifferenced analysis algorithm was presented. We continued to observe in a lead trail or LMT pattern in 2005 in order to produce maps wider than the maximum range $\left(\sim 3^{\circ}\right)$ of the chopping tertiary mirror. The undifferenced analysis presented in K07, and used for this paper's analysis, is outlined below with any differences in the application to the 2005 data set highlighted.

Let $d_{\alpha}$ be a measurement of the CMB temperature at pixel $\alpha$. The data vector can be represented as the sum of the signal, noise, and chopper synchronous offsets: $d_{\alpha}=s_{\alpha}+n_{\alpha}+o_{\alpha}$. For example, although the chopping mirror moves the beams at nearly constant elevation, the slight residual atmospheric gradient produces a chopper synchronous signal $o_{\alpha}$, which is an approximately quadratic function of chopper angle. To remove these offsets, the data from each chopper sweep are filtered with the "corrupted mode projection" matrix $\Pi$ to produce the cleaned time stream $\tilde{\mathbf{d}} \equiv \Pi \mathbf{\Pi d}$.

The $\Pi$ matrix projects a third- to tenth-order polynomial, which suppresses large angular scale chopper offsets. The order of the polynomial removed depends on the amplitude of atmosphere-induced cross-channel correlations. As described in K07, small angular scale offsets can be removed by subtracting an "average" chopper function. For 2002 data, we removed a chopper synchronous offset from each data strip where the amplitude of the offset at each sample in the strip is free to vary quadratically with elevation in the map. The large fields observed in 2005 have up to four times the decl. range of the fields observed in 2001 and $2002\left(\sim 10^{\circ}\right.$ versus $\left.\sim 2.5\right)$. For 2005 data, we allow the offset to vary from a third to fifth order polynomial depending on the extent of the map in declination. A zeroth-order polynomial in elevation removes the average chopper function and the higher-order terms effectively act as a high-pass filter on changes in the offset as a function of time or elevation. This anisotropic filtering removes offset-corrupted modes while preserving most of the uncorrupted modes for the power spectrum analysis. The loss of information at high $\ell$ is small; the removed modes account for only a few percent of the total degrees of freedom of the data.

The corrupted mode projection matrix $\Pi$ can be represented as the product of two matrices, $\Pi \equiv \Pi_{2} \Pi_{1}$. The operator $\Pi_{1}$ is the original $\Pi$ matrix referenced in $\mathrm{K} 04$ which adaptively removes polynomial modes in R.A. The additional operator $\Pi_{2}$ removes modes in decl. independently for each of the lead, main, and trail fields and can be further decomposed into the product $\Pi_{2}=\Pi_{2}^{\text {Poly }} \Pi_{2}^{\mathrm{LPF}}$. The operator $\Pi_{2}^{\text {Poly }}$ performs the aforementioned polynomial projection in decl. to remove smallscale chopper offsets. The second operator $\Pi_{2}^{\mathrm{LPF}}$ imposes a low- pass filter (LPF) $\ell<3200$ on each decl. strip. The decl. strips are perpendicular to the scan direction; the time streams have always had an LPF applied in the scan direction. The pixelation used when estimating the power spectrum is too large to resolve all of the noise power (at $\ell$ up to 10,800 ), causing out-of-band noise to be aliased into the signal band $(\ell<3000)$ if an LPF is not applied. Eliminating this high-frequency noise reduces the contribution of instrumental noise to the reported band powers.

Using the pointing model, the cleaned time streams are coadded to create a map:

$$
\Delta=\mathbf{L d}
$$

The noise covariance matrix of the map can be represented as

$$
\mathbf{C}_{N}=\mathbf{L}\left\langle\mathbf{n n}^{t}\right\rangle \mathbf{L}^{t}
$$

where $\mathbf{n}$ is the time stream noise. The noise matrix is diagonalized as part of applying a high signal-to-noise transformation to the data. Eliminating modes with insignificant information content reduces the computational requirements of later steps in the analysis.

In order to apply the iterative quadratic band-power estimator, we need to know the partial derivative $\frac{\partial \mathbf{C}_{T}}{\partial q_{B}}$ of the theory covariance matrix $\mathbf{C}_{T}$ with respect to each band-power $q_{B}$. The theory matrix can be calculated by considering the effects of the filtering on the raw sky signal. The signal time stream $s_{\alpha}$ is the convolution of the true temperature map $\mathfrak{T}(\mathbf{r})$ with the instrumental beam function $B_{\alpha}(\mathbf{r})$

$$
s_{\alpha}=\int d^{2} r \mathfrak{T}(\mathbf{r}) B_{\alpha}(\mathbf{r}) .
$$

The signal component of the coadded map will be $\Delta^{\text {sig }}=\mathbf{L s}$ or

$$
\Delta_{i}^{\mathrm{sig}}=\int d^{2} r F_{i}(\mathbf{r}) \mathfrak{T}(\mathbf{r})
$$

where we have defined the pixel-beam filter function $F_{i}$

$$
F_{i}(\mathbf{r})=\sum_{\alpha} L_{i \alpha} B_{\alpha}(\mathbf{r})
$$

The theory covariance matrix can be calculated in the flat sky case to be

$$
\begin{aligned}
C_{T\{i j\}} & \equiv\left\langle\Delta_{i} \Delta_{j}\right\rangle^{\mathrm{sig}}=\iint d^{2} r d^{2} r^{\prime} F_{i}(\mathbf{r}) F_{j}\left(\mathbf{r}^{\prime}\right)\left\langle\mathfrak{T}(\mathbf{r}) \mathfrak{T}\left(\mathbf{r}^{\prime}\right)\right\rangle \\
& =\iint d^{2} r d^{2} r^{\prime} F_{i}(\mathbf{r}) F_{j}\left(\mathbf{r}^{\prime}\right) \int \frac{d^{2} \ell}{(2 \pi)^{2}} C_{\ell} \cdot e^{i \mathbf{l} \cdot\left(\mathbf{r}-\mathbf{r}^{\prime}\right)} \\
& =\int \frac{d^{2} \ell}{(2 \pi)^{2}} C_{\ell} \cdot \tilde{F}_{i}^{*}(\mathbf{I}) \tilde{F}_{j}(\mathbf{l}),
\end{aligned}
$$

where $\tilde{F}_{i}(\mathbf{l})$ is the Fourier transform of the pixel-beam filter function. The partial derivative of the theory matrix can be calculated in a straightforward manner from Equations (1) and (2).

This algorithm does not require the instrument beams to remain constant. The actual ACBAR beam sizes vary slightly with chopper angle (Runyan et al. 2003). The measured beam variations can be fit to a semianalytic function as described in K04 to create a more accurate representation of the true beam shape across the map. We use the corrected beam sizes when removing point sources. In K04 and K07, we found that the 
Table 2

Millimeter Bright PMN Sources

\begin{tabular}{|c|c|c|c|c|}
\hline Source Name/Position & Field & $S_{4.85}(\mathrm{mJy})$ & $S_{150}(\mathrm{mJy})$ & $\alpha_{150 / 4.85}$ \\
\hline PMN J0455-4616* & CMB2 & 1653 & $2898 \pm 60$ & 0.16 \\
\hline PMN J0439-4522 & CMB2 & 634 & $383 \pm 73$ & -0.15 \\
\hline PMN J0451-4653 & CMB2 & 541 & $360 \pm 58$ & -0.12 \\
\hline PMN J0253-5441* & CMB5 & 1193 & $1260 \pm 62$ & 0.02 \\
\hline PMN J0223-5347 & CMB5 & 397 & $176 \pm 27$ & -0.24 \\
\hline PMN J0229-5403 & CMB5 & 242 & $147 \pm 17$ & -0.14 \\
\hline PMN J0210-5101* $*^{\circ}$ & CMB6 & 3198 & $1268 \pm 86$ & -0.27 \\
\hline PMN J2207-5346 $*^{\circ}$ & CMB7ext & 1410 & $381 \pm 68$ & -0.38 \\
\hline PMN J2235-4835* $*^{\circ}$ & CMB7ext & 1104 & $1509 \pm 75$ & 0.09 \\
\hline PMN J2239-5701* & CMB7ext & 1063 & $501 \pm 68$ & -0.22 \\
\hline PMN J2246-5607 & CMB7ext & 618 & $386 \pm 51$ & -0.14 \\
\hline PMN J2309-5703 & CMB7ext & 56 & $257 \pm 76$ & 0.44 \\
\hline PMN J0519-4546* $*^{\circ}$ & CMB8 & 15827 & $1375 \pm 101$ & -0.71 \\
\hline PMN J0519-4546* & CMB8 & 14551 & $1148 \pm 86$ & -0.74 \\
\hline PMN J0538-4405* $*^{\circ}$ & CMB8 & 4805 & $7114 \pm 87$ & 0.11 \\
\hline PMN J0515-4556 $*^{\circ}$ & CMB8 & 990 & $671 \pm 96$ & -0.11 \\
\hline PMN J0526-4830 & CMB8 & 425 & $82 \pm 25$ & -0.48 \\
\hline PMN J0525-4318 & CMB8 & 217 & $99 \pm 25$ & -0.23 \\
\hline PMN J0531-4827 & CMB8 & 142 & $96 \pm 25$ & -0.11 \\
\hline PMN J2357-5311 $*^{\circ}$ & CMB9 & 1782 & $347 \pm 49$ & -0.48 \\
\hline PMN J2336-5236 & CMB9 & 1588 & $233 \pm 57$ & -0.56 \\
\hline PMN J2334-5251 & CMB9 & 557 & $432 \pm 57$ & -0.07 \\
\hline PMN J0018-4929 & CMB9 & 142 & $178 \pm 57$ & 0.07 \\
\hline PMN J0026-5244 & CMB9 & 40 & $192 \pm 62$ & 0.46 \\
\hline PMN J0050-5738 $*^{\circ}$ & CMB10 & 1338 & $773 \pm 108$ & -0.16 \\
\hline PMN J0058-5659 $*^{\circ}$ & CMB10 & 739 & $514 \pm 61$ & -0.11 \\
\hline PMN J0133-5159*º & CMB10 & 672 & $248 \pm 72$ & -0.29 \\
\hline PMN J0124-5113 $*^{\circ}$ & CMB10 & 308 & $335 \pm 48$ & 0.02 \\
\hline PMN J2208-6404 & CMB11 & 53 & $136 \pm 44$ & 0.27 \\
\hline PMN J0103-6438 & CMB12 & 395 & $268 \pm 65$ & -0.11 \\
\hline PMN J0144-6421 & CMB12 & 152 & $184 \pm 59$ & 0.06 \\
\hline PMN J0303-6211*º & CMB13 & 1862 & $423 \pm 63$ & -0.43 \\
\hline PMN J0309-6058 $*^{\circ}$ & CMB13 & 1103 & $596 \pm 80$ & -0.18 \\
\hline PMN J0251-6000 & CMB13 & 433 & $189 \pm 34$ & -0.24 \\
\hline PMN J0236-6136 & CMB13 & 406 & $365 \pm 33$ & -0.03 \\
\hline PMN J0257-6112 & CMB13 & 178 & $104 \pm 33$ & -0.16 \\
\hline PMN J0231-6036 & CMB13 & 174 & $105 \pm 33$ & -0.15 \\
\hline
\end{tabular}

Notes. These sources from the PMN $4.85 \mathrm{GHz}$ catalog are detected at $>3.0 \sigma$ significance with ACBAR, corresponding to a false detection rate of $<2.2$. The fluxes at $4.85 \mathrm{GHz}\left(S_{4.85}\right.$, from Wright et al. 1994) and $150 \mathrm{GHz}\left(S_{150}\right.$, measured by ACBAR) are given. For ACBAR, the flux conversion factor is $1 \mu \mathrm{K}_{\mathrm{CMB}}=0.9 \mathrm{mJy}$. The spectral index $\alpha$ is defined as $S_{v} \propto v^{\alpha}$. The flux of some of these sources varied by up to $50 \%$ between years; this variability is not reflected in the estimated errors. The central guiding quasars (one in each of the five deeper fields) are marked with asterisks $\left(^{*}\right)$. These sources, as well as all other PMN sources $>40 \mathrm{mJy}$, are projected from the data using the methods described by $\mathrm{K} 04$, and do not contribute to the power spectrum results. The brightest sources are marked with circles $\left({ }^{\circ}\right)$ and are removed from the maps in a beam-independent method. Note that PMN J0519-4546a/b are within one beam width of each other and are not separately resolved by ACBAR. As a result, the listed $\alpha$ for PMN J0519-4546a/b is estimated from the sum of the fluxes at $4.85 \mathrm{GHz}$ and the mean of the fluxes at $150 \mathrm{GHz}$.

differences in the power spectra from using the map-averaged beam or exact beam for each pixel were negligible. For the band powers reported in Table 3, an averaged beam is used for the entire map.

As in K07, we calculate the full two-dimensional noise correlation matrix directly from the time stream data without using Fourier transforms. All the numerical calculations are performed on the National Energy Research Scientific Computing Center (NERSC) IBM SP RS/6000. The evaluation of the Fourier transform of $F_{i}(\mathbf{r})$ is the most computationally expensive step of
Table 3

Joint Likelihood Band Powers

\begin{tabular}{|c|c|c|c|c|}
\hline$\ell$ range & $\ell_{\mathrm{eff}}$ & $q\left(\mu \mathrm{K}^{2}\right)$ & $\sigma\left(\mu \mathrm{K}^{2}\right)$ & $\mathbf{x}\left(\mu \mathbf{K}^{2}\right)$ \\
\hline $350-550$ & 470 & 2250 & 92 & -345 \\
\hline $550-650$ & 608 & 1982 & 92 & -303 \\
\hline $650-730$ & 694 & 1879 & 89 & -267 \\
\hline $730-790$ & 763 & 2180 & 111 & -239 \\
\hline $790-850$ & 823 & 2391 & 115 & -255 \\
\hline $850-910$ & 884 & 1824 & 90 & -164 \\
\hline $910-970$ & 944 & 1427 & 70 & -104 \\
\hline $970-1030$ & 1003 & 1111 & 57 & -18 \\
\hline $1030-1090$ & 1062 & 1043 & 54 & 17 \\
\hline $1090-1150$ & 1122 & 1143 & 57 & 34 \\
\hline $1150-1210$ & 1182 & 1067 & 54 & 75 \\
\hline $1210-1270$ & 1242 & 808 & 46 & 119 \\
\hline $1270-1330$ & 1301 & 693 & 43 & 154 \\
\hline $1330-1390$ & 1361 & 778 & 47 & 193 \\
\hline $1390-1450$ & 1421 & 746 & 46 & 218 \\
\hline $1450-1510$ & 1481 & 604 & 44 & 241 \\
\hline $1510-1570$ & 1541 & 517 & 41 & 229 \\
\hline $1570-1650$ & 1618 & 435 & 34 & 261 \\
\hline $1650-1750$ & 1713 & 363 & 30 & 242 \\
\hline $1750-1850$ & 1814 & 344 & 32 & 264 \\
\hline $1850-1950$ & 1898 & 227 & 33 & 170 \\
\hline $1950-2100$ & 2020 & 217 & 31 & 203 \\
\hline $2100-2300$ & 2194 & 162 & 31 & 244 \\
\hline $2300-2500$ & 2391 & 159 & 43 & 357 \\
\hline $2500-3000$ & 2646 & 105 & 45 & 560 \\
\hline
\end{tabular}

Notes. Band multipole range and weighted value $\ell_{\text {eff }}$, decorrelated band powers $q_{B}$, uncertainty $\sigma_{B}$, and lognormal offset $x_{B}$ from the joint likelihood analysis of the 10 ACBAR fields. The positive trend in the lognormal offsets with increasing $\ell$ is due to the increasing contribution of instrumental noise to the error budget; the lognormal offset approaches infinity in the Gaussian limit. The PMN radio point source and IRAS dust foreground templates have been projected in this analysis.

this analysis. We use an iterative quadratic estimator to find the maximum likelihood band powers (Bond et al. 1998). The resulting band powers are presented in Table 3 and Figure 5.

\section{CALIBRATION}

We derive the absolute calibration of ACBAR by directly comparing the 2005 ACBAR maps to the WMAP5 V and Wband temperature maps (Hinshaw et al. 2008). We pass the WMAP5 maps through a simulated version of the ACBAR pipeline to ensure equivalent filtering and cross-spectra are calculated for each field. The ratios of the cross-spectra are used to measure the relative calibration after being corrected for the respective instrumental beam functions. We had initially applied this calibration scheme to the WMAP3 maps. Transitioning to the WMAP5 data set lowered the calibration by $1.4 \%$ in CMB temperature units and slightly reduced the overall uncertainty. The ACBAR band powers are unchanged except for this calibration factor. Results for ACBAR's six largest fields (approximately $600 \mathrm{deg}^{2}$ in area) are combined to achieve a calibration accuracy of $1.97 \%$ for the 2005 data.

The 2005 calibration is transferred to 2001 and 2002 through a comparison of power spectra for overlapping regions observed by ACBAR in each year. The CMB5 field is used to extend the calibration of the 2005 season to the 2002 data. The CMB5 calibration is carried to other fields observed in 2002 by daily observations of the flux of RCW38. The calibration of the CMB4 field (observed in 2002) is then transferred to the $70 \%$ 


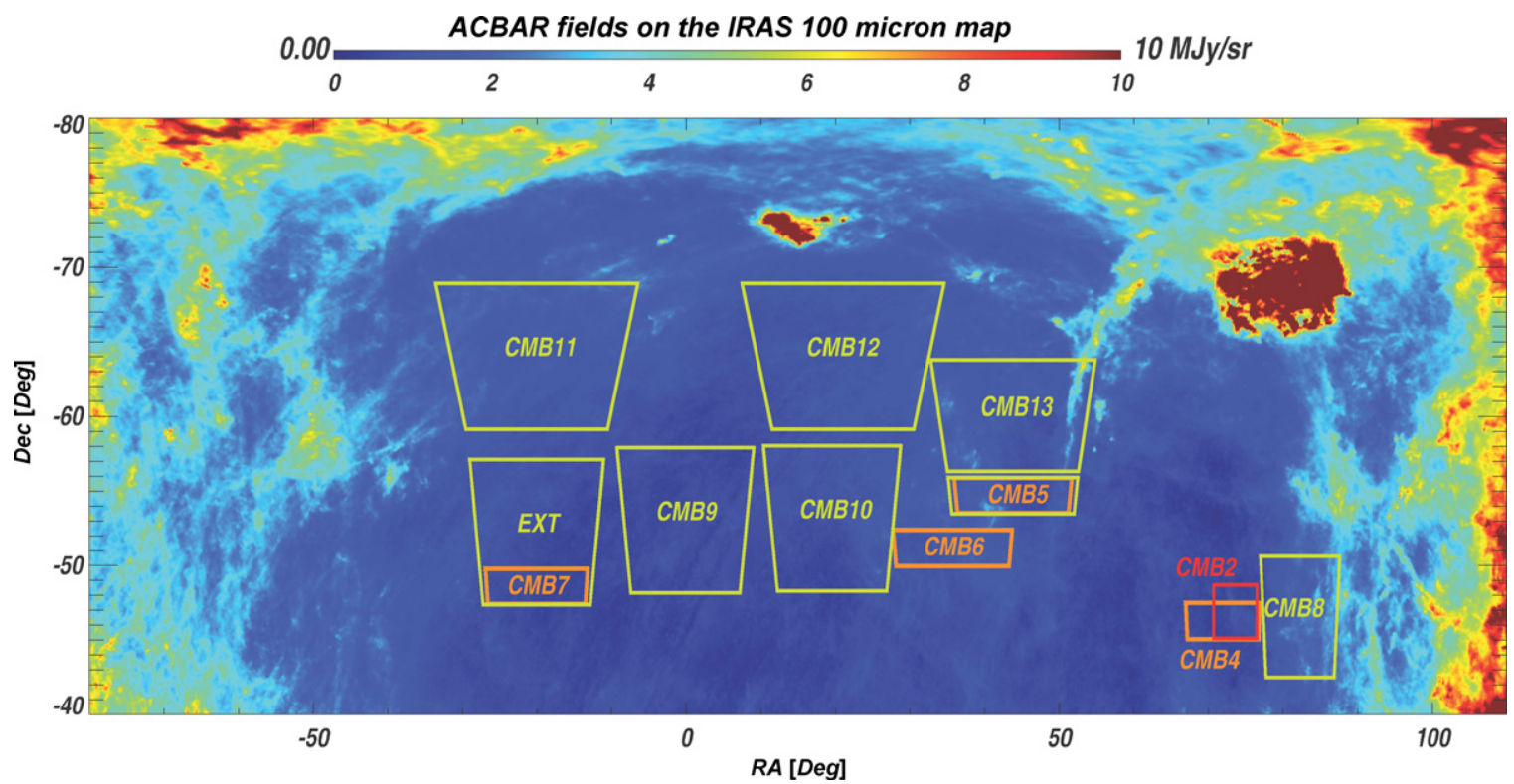

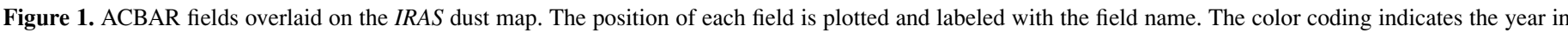

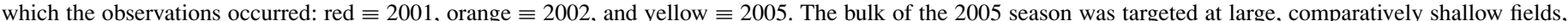

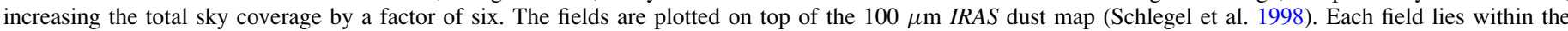

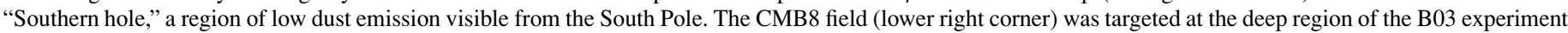
as an alternative calibration path to the WMAP cross-calibration used for the results presented here.

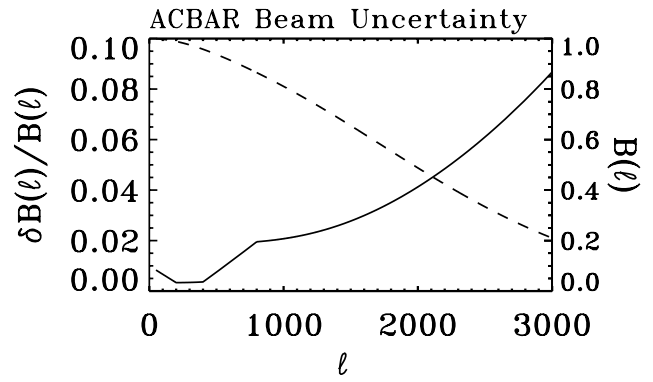

Figure 2. ACBAR beam uncertainty and beam function. Solid line and left axis: the $1 \sigma$ envelope for uncertainty in the ACBAR beam function $B_{\ell}$. The increasing uncertainty above $\ell=1000$ reflects the $2.6 \%$ uncertainty in the fitted Gaussian FWHMs. The behavior at $\ell<1000$ is a combination of the uncertainty in the measured sidelobes and the calibration method "pinning" the transfer function for $\ell \in[256,512]$. Dashed line and right axis: the measured ACBAR beam function.

overlapping CMB2 field (observed in 2001). According to the new calibration, results from the RCW38-based calibration used for the 2002 data in K07 need to be multiplied by $0.959 \pm 0.032$. Including the year-to-year calibration uncertainty, the final calibration has an uncertainty of $2.05 \%$ in $\mathrm{CMB}$ temperature units $(4.1 \%$ in power). Additional details of this procedure are discussed in the Appendices.

\section{BEAM DETERMINATION}

The beams are well described by a symmetric Gaussian, with their main-lobe FWHM determined to $2.6 \%$ by continuous measurements of the images of bright quasars located in the CMB fields. The beam sidelobes were measured to the level of $30 \mathrm{~dB}$ with observations of Venus made in 2002. Venus is extremely bright at millimeter wavelengths and with a diameter of $\lesssim 1^{\prime}$ is much smaller than ACBAR's beam size. However, there are extended periods during which ACBAR was unable to observe Venus. ACBAR observed RCW38, a bright $\mathrm{H}$ II region in the galactic plane, every day. We compare deep, coadded observations of RCW38 to constrain the temporal variability of the beam sidelobes when Venus was unavailable. The complex structure surrounding RCW38 makes it difficult to directly recover the beam shape $B(\mathbf{r})$. Instead, we monitor ratios of the beam-smoothed RCW38 maps $\int d^{2} r S^{\mathrm{RCW} 38}(\mathbf{r}) B(\mathbf{r})$. Any observed differences in the maps would indicate a change to the instrumental beam function as the morphology of RCW38's emission $S^{\mathrm{RCW} 38}$ is expected to be constant. We set an upper limit on the possible temporal variations in the map and use this to constrain temporal variations in the beam function. The estimated band-power uncertainty from the beam function is comparable to the overall calibration uncertainty and is plotted in Figure 2.

\section{SYSTEMATIC UNCERTAINTIES AND FOREGROUNDS}

\subsection{Jackknife Tests}

We performed a series of tests to search for and constrain potential systematic errors in the power spectrum results. As described in $\mathrm{K} 04$, the data can be divided into two halves based on whether the chopping mirror is moving to the left or right. The "left minus right" jackknife is a sensitive test for errors in the transfer function correction, microphonic vibrations excited by the chopper motion, or the effects of wind direction. Maps with bright sources such as RCW38 can provide particularly sensitive tests of the transfer function (see Runyan et al. 2003, for a description of ACBAR's transfer functions). Similarly, the data can be split based on the time that the observation occurred. A nonzero signal could be produced in the "first half minus second half" jackknife by variation in the calibration, pointing, beam and sidelobe, or any other time-dependent variations in the instrument. In addition, the band powers of each jackknife constrain the mis-estimation of noise during that period.

We applied the left-right jackknife to the $2005 \mathrm{CMB}$ data and found the band powers were inconsistent with zero at $2.5 \sigma$ at high $\ell(\ell>2100)$. We reran a set of left-right jackknives, 


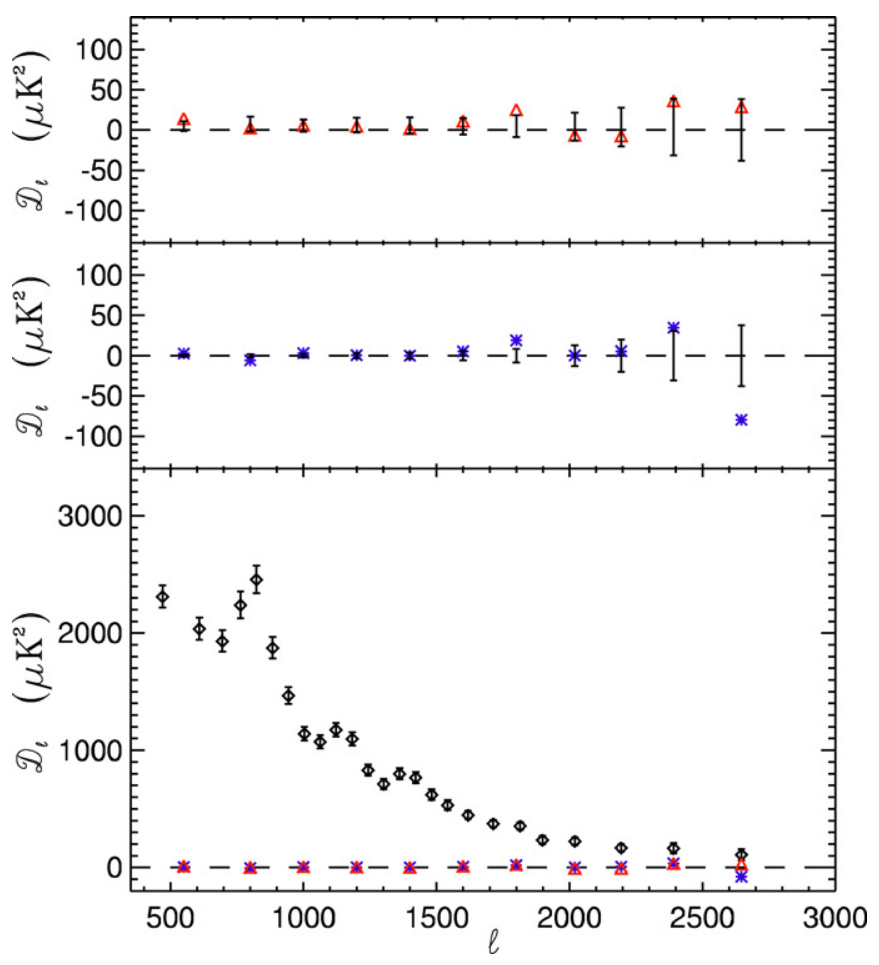

Figure 3. Systematic tests performed on the ACBAR data. Top: power spectrum (red triangle) for differenced maps from the first half and second half of the season for each field, compared to the results of Monte Carlo simulations (error bars). Middle: power spectrum (the blue star) derived from difference maps of the left- and right-going chopper sweeps for all ten fields. Bottom: the undifferenced band powers from Table 3 (black diamonds) compared to both jackknife power spectra: the left-right jackknife (the blue star) and first half-second half jackknife (red triangle).

dropping individual channels, and found that two channels stood out. With both channels excluded, the discrepancy in the leftright jackknife band powers disappeared. We were unable to find evidence for unusual microphonic lines or transfer functions in the two problematic channels, but hypothesize that these two channels have subtle microphonic response in the signal bandwidth that are detectable only in a deep integration. Both channels are excluded from the 2005 data for all results reported in this paper.

We apply the first-second half jackknife test to the joint CMB power spectrum with the exclusion of the bad channels from the 2005 data. The power spectrum of the chronologically differenced maps is compared to the band powers of a set of Monte Carlo realizations of simulated difference maps in order to account for a number of effects, such as the small filtering differences due to different scan patterns and the temporal uncertainty in the beam sidelobes (see Section 5). We find that the jackknife band powers are consistent with the predictions of the Monte Carlo above $\ell=400$. There is a $4 \sigma$ residual of $\sim 15 \mu \mathrm{K}^{2}$ in the first bin. Because the combined statistical and cosmic variance uncertainty in this bin is a factor of six larger, we assume that the band-power estimate will not be significantly biased.

We also perform the left-right jackknife on the joint $\mathrm{CMB}$ power spectrum found from the complete data set. The results are consistent with zero for $\ell>900$. Statistically, the probability to exceed the measured $\chi^{2}$ for $\ell>900$ is $15 \%$. The results are inconsistent with zero at a very low $\left(\sim 4 \mu \mathrm{K}^{2}\right)$ level (Figure 3 ) on larger angular scales. This residual could be due to a small noise mis-estimate at low $\ell$, possibly caused by neglected atmospheric correlations. The jackknife failure of $\sim 4 \mu \mathrm{K}^{2}$ is much smaller than the band-power uncertainties (90-300 $\mu \mathrm{K}^{2}$ ) in these $\ell$ bins, which are dominated by cosmic variance. The first-second half jackknife is insensitive to discrepancies of this magnitude due to the greater uncertainties introduced by small pointing and filtering differences. We conclude that the complete ACBAR data set shows no significant residuals in the jackknife tests, and we expect no significant systematic contamination of the resulting power spectrum.

\subsection{Foregrounds}

The potential contribution of foreground emission must be considered in the interpretation of $\mathrm{CMB}$ temperature anisotropies. There are three foregrounds with significant emission at $150 \mathrm{GHz}$ on the relevant angular scales: galactic dust, extragalactic radio sources, and dusty protogalaxies. As an effectively single-frequency instrument, ACBAR depends on data from other experiments to construct and constrain foreground models. We use the methodology described in K04 to remove templates for radio sources and dust emission from the CMB maps without making assumptions about their flux. The contribution from dusty protogalaxies is less certain; however, we do not expect the combined residual foreground emission to significantly impact the power spectrum for $\ell<2400$.

We remove modes from the $\mathrm{CMB}$ maps corresponding to radio sources in the $4.85 \mathrm{GHz}$ Parkes-MIT-NRAO (PMN) survey (Wright et al. 1994). Of the 1601 PMN sources with a flux greater than $40 \mathrm{mJy}$ that lie in the ACBAR fields, we detect 37 sources including the guiding quasars at greater than $3 \sigma$ with the application of an optimal-matched filter. There are less than 2.2 false detections expected with this detection threshold. The measurement errors are estimated through sampling the distribution of pixels in a set of 100 Monte Carlo realizations of the $\mathrm{CMB}+$ noise for each field. Table 2 lists the parameters of the detected PMN sources. Except for the few bright sources detected at $150 \mathrm{GHz}$, removing the PMN point sources does not significantly affect the band powers.

It is possible that faint radio sources, undetected at $150 \mathrm{GHz}$, could contribute to the observed band powers. We parameterize the contribution as

$$
\mathcal{D}_{\ell}^{\mathrm{src}}=q_{\mathrm{src}}\left(\frac{\ell}{2600}\right)^{2} \mu \mathrm{K}^{2},
$$

which is appropriate for unclustered point sources. We compare the $150 \mathrm{GHz}$ ACBAR point source number counts to the model in White \& Majumdar (2004), based on WMAP $Q$-band data,

$$
\frac{d N}{d S_{v}}=\frac{80 \mathrm{deg}^{-2}}{1 \mathrm{mJy}}\left(\frac{S_{v}}{1 \mathrm{mJy}}\right)^{-2.3},
$$

to constrain the residual power contribution at $150 \mathrm{GHz}$. We can use this model to estimate the residual band-power contribution due to sources too faint to be included in the PMN catalog.

Following the convention in that work, the spectral dependence of the fluxes is parameterized as $S_{v} \propto v^{\beta}$. The number of PMN sources detected at $150 \mathrm{GHz}$ in a logarithmic flux bin, $n_{B}^{\text {obs }}$, are compared to the predicted number counts from the same population of sources with a given $\beta, n_{B}^{(\beta)}+n_{B}^{\text {noise }}$. Here, $n_{B}^{(\beta)}$ is the modeled number counts and $n_{B}^{\text {noise }}$ is the expected number of false detections due to ACBAR's measurement uncertainty. The number counts are assumed to follow a Poisson distribution. Sources with estimated measurement errors greater than $140 \mathrm{mJy}$ in the ACBAR maps are cut to reduce 
the $n_{B}^{\text {noise }}$ term. The modeled number counts $n_{B}^{(\beta)}$ are scaled by $\left(N_{\text {tot }}-N_{\text {cut }}\right) / N_{\text {tot }}$ to compensate. All other sources with measured amplitudes greater than $350 \mathrm{mJy}$ at $150 \mathrm{GHz}$ are included in the calculation without consideration of the signal to noise. If the sources found by ACBAR at $150 \mathrm{GHz}$ are the same population found by WMAP, this implies a uniform spectral index of $\beta=0.14 \pm 0.15$. However, this small sample selected for high flux at $150 \mathrm{GHz}$ is heavily biased toward sources with flat or rising spectra. We increase ACBAR's sensitivity to dimmer sources by binning all sources within a given PMN flux range, and look at the ratio of the average flux at $150 \mathrm{GHz}$ to the average flux at $4.85 \mathrm{GHz}$ within each bin. We find the ratio $\left(S_{150} / S_{4.85}\right)$ increases with PMN flux from 0.07 for sources below $400 \mathrm{mJy}$ to 0.41 for sources above $1600 \mathrm{mJy}$ at $4.85 \mathrm{GHz}$. This implies that the sources in the PMN catalog have a flux-dependent spectral index where dimmer objects typically have a more steeply falling spectrum. The band-power contribution of the low-flux sources depends sensitively on the extrapolation of the $40 \mathrm{mJy}$ flux cutoff in the $4.85 \mathrm{GHz}$ PMN catalog to $150 \mathrm{GHz}$. Based on the observed flux ratios for PMN sources with $S_{4.85}<400 \mathrm{mJy}$, we conservatively assume $S_{150} / S_{4.85}=0.1$ for a flux cutoff at $4 \mathrm{mJy}$ at $150 \mathrm{GHz}$. This flux ratio corresponds to a spectral index of $\beta=-0.67$, well below $\beta=0.14 \pm 0.15$ estimated from the ACBAR detected sources and the WMAP $Q$-band source model. Estimating the residual radio source band-power contribution at $150 \mathrm{GHz}$ with a flux cutoff of $4 \mathrm{mJy}$ gives $q_{\mathrm{src}}^{\text {radio }}=2.2$. At this level, the residual contribution from radio sources will be negligible in the ACBAR data.

The ACBAR fields are positioned in the "Southern Hole," a region of exceptionally low Galactic dust emission (Figure 1). Finkbeiner et al. (1999, FDS99) constructed a multicomponent dust model that predicts thermal emission at $\mathrm{CMB}$ frequencies from the combined observations of IRAS, COBE/DIRBE, and COBE/FIRAS. Taking into account the ACBAR filtering, the FDS99 model $^{13}$ predicts an RMS dust signal at the few $\mu \mathrm{K}$ level, primarily on large angular scales. The ACBAR maps can be decomposed as the sum of the CMB and dust signals $T_{\mathrm{CMB}}+\xi T_{\mathrm{FDS}}$. The dust amplitude parameter $\xi$ is predicted to equal unity by the FSD99 model. The ACBAR maps are cross-correlated with the dust templates $T_{\mathrm{FDS}}$ to calculate the amplitude in each field. The errors are estimated by applying the same procedure to $100 \mathrm{CMB}+$ noise map realizations for each field. The uncertainty in $\xi$ is dominated by CMB fluctuations. The best-fit amplitude from combining all the fields is $\xi=$ $0.1 \pm 0.5$. The estimated amplitudes of the individual fields are shown in Figure 4. The reduced $\chi^{2}$ of the measured amplitudes $\xi \mathrm{s}$ of the eight fields analyzed is 0.75 for the no-dust assumption of $\langle\xi\rangle=0$ and increases to $\chi^{2}=1.12$ for the FDS99 model amplitude of $\langle\xi\rangle=1$. Therefore, the ACBAR data slightly favor a lower amplitude than predicted by the FDS99 model. The dust signal is not detectable in any of the ACBAR fields, and removing the dust template has a negligible impact on the measured power spectrum.

Dusty IR galaxies are the third and least-constrained foreground in the ACBAR fields. This population of high redshift, star-forming galaxies has been studied by several experiments at higher frequencies (Coppin et al. 2006; Laurent et al. 2005; Maloney et al. 2005; Greve et al. 2004). However, as discussed in $\mathrm{K} 07$, extrapolating the expected signal to $150 \mathrm{GHz}$ remains highly uncertain, and there remain significant uncertainties in the number counts $d N / d S$ and spatial clustering of the sources.

\footnotetext{
13 We use the default model 8 of FDS99.
}

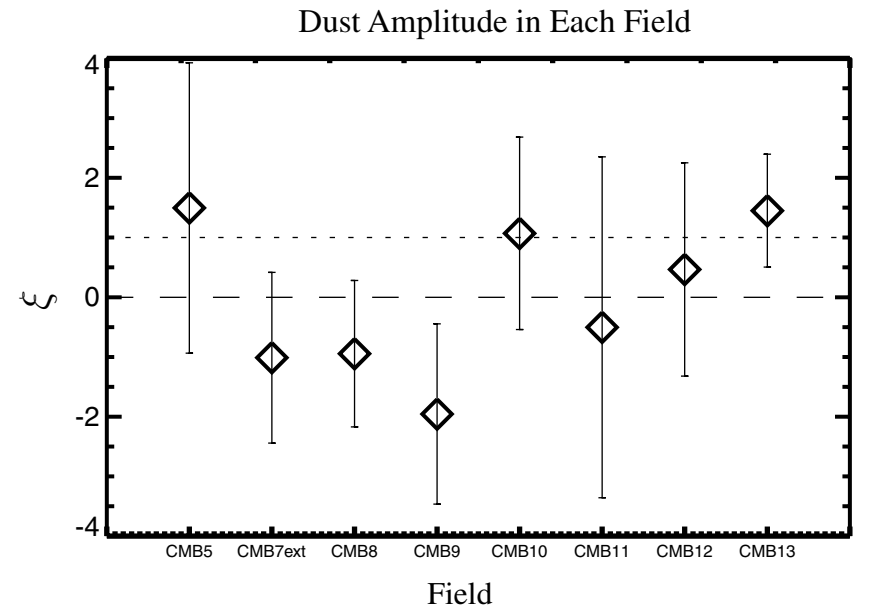

Figure 4. Dust emission is not detected in the ACBAR fields. Parameterizing the dust signal as $T_{\mathrm{CMB}}+\xi T_{\mathrm{FDS}}$, a suite of Monte Carlo realizations of maps of $\mathrm{CMB}$ and noise is used to estimate the uncertainty in $\xi$. We find the upper limits in each field to be consistent with the FDS99 model $(\xi=1)$, but the data somewhat favor a lower dust amplitude. The reduced $\chi^{2}$ of the measured amplitudes $\xi \mathrm{s}$ is 0.75 under the assumption that $\langle\xi\rangle=0$ (the dashed line). The reduced $\chi^{2}$ for the FDS99 model with $\langle\xi\rangle=1$ is 1.12 (the dotted line).

The frequency dependence can be empirically determined by comparing the measured number counts in overlapping fields observed at different frequencies. This comparison has been done with MAMBO $(1.2 \mathrm{~mm})$ and SCUBA $(850 \mu \mathrm{m})$, leading to a spectral dependence of $S_{v} \propto v^{2.65}$ (Greve et al. 2004). A second method for estimating the index used nearby galaxy data to obtain $S_{v} \sim v^{2.6 \pm 0.3}$ (Knox et al. 2004). The uncertainty in the spectral dependence significantly affects the extrapolation of the flux of dusty galaxies to $150 \mathrm{GHz}$. We use estimates of the source number counts from the SHADES survey (Coppin et al. 2006) and Bolocam Lockman Hole Survey (Maloney et al. 2005). We apply the formulas in Scott \& White (1999) to estimate the expected power spectrum for the source number counts, ignoring the clustering terms. In this limit, $\mathcal{D}_{\ell}$ will have the form in Equation (3). Scaling the results to $150 \mathrm{GHz}$ with the MAMBO/SCUBA prescription of $S_{v} \propto v^{2.65}$ leads to an estimated contribution of $q_{\text {src }}^{\text {dusty }}=17-29$. This range reflects the differences between the measured number counts, but does not include the uncertainty in the spectral dependence of the fluxes. Combining the median index with earlier SCUBA data fit by two power laws in $S$ from (Borys et al. 2003), we find an excess of $22 \mu \mathrm{K}^{2}$ at $\ell=2600$, within that range. This level is only a factor of two smaller than the instrumental noise of ACBAR and might influence the interpretation of high $\ell$ band powers. We tentatively assume that contamination from dusty protogalaxies does not significantly affect the resulting power spectrum. The implications of relaxing this assumption for cosmological parameter estimation are explored in Section 8.3.

\section{RESULTS AND DISCUSSIONS}

\subsection{Power Spectrum}

The power spectrum presented in Figure 5 is produced by the application of the analysis algorithm outlined in Section 3 to ACBAR data from the 2001, 2002, and 2005 austral winters. The resulting power spectrum is compared to the WMAP5 and B03 spectra in Figure 6. The zero curvature, $\Lambda \mathrm{CDM}$ "ACBAR+WMAP5" best fit model, is shown in each figure for reference. The decorrelated band powers are tabulated 


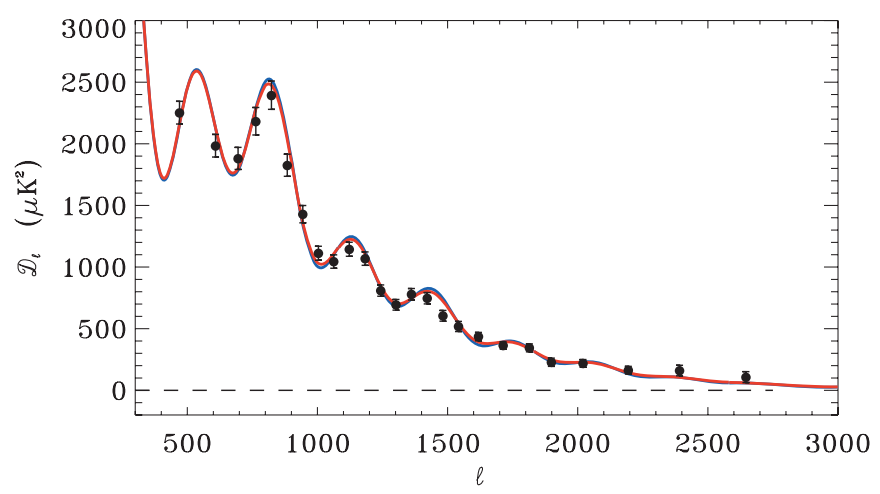

Figure 5. Decorrelated ACBAR band powers for the full data set. The $1 \sigma$ error bars are derived from the offset lognormal fits to the likelihood function. The band powers are in excellent agreement with an $\Lambda$ CDM model. The damping of the anisotropies is clearly seen with an $\mathrm{S} / \mathrm{N}>4$ out to $\ell=2500$. The third acoustic peak (at $\ell \sim 800$ ), fourth acoustic peak (at $\ell \sim 1100$ ), and fifth acoustic peak (at $\ell \sim 1400$ ) are visible. The plotted lines are the best fits to the ACBAR and WMAP5 band powers for a spatially flat, $\Lambda$ CDM universe with no SZE contribution. A lensed (red) and unlensed (blue) model spectrum is shown for a fixed parameter set; the lensed spectrum is a significantly better fit to the ACBAR data



Figure 6. ACBAR band powers plotted with those from WMAP5 (Hinshaw et al. 2008) and the 2003 flight of BOOMERANG (Jones et al. 2006). The three experiments show an excellent agreement in the region where they overlap.

in Table 3. Our choice of the decorrelation transformations follows Tegmark (1997). The band powers can be compared to a theoretical model using the window functions (Knox 1999). As in K04, we sample the likelihood function $\mathcal{L}(\Delta)=$ $\frac{1}{\sqrt{C}} e^{-\left(\Delta^{t} C^{-1} \Delta\right) / 2}$ near the maximum and fit the results with offset lognormal functions (Bond et al. 2000). The fit parameters $\sigma, \mathbf{x}$ are listed in Table 3 as well. The band powers, likelihood-fit parameters, and window functions are available for download from the ACBAR website. ${ }^{14}$

The ACBAR data extend the measurement of the temperature anisotropies well into the damping tail with $\mathrm{S} / \mathrm{N}>5$ for $\ell \lesssim 2300$. The fourth and fifth acoustic peaks are detected for the first time in the ACBAR band powers, providing additional support for the coherent origin of anisotropy (Albrecht et al. 1996). The position of the third acoustic peak is consistent with previous detections of the feature by $\mathrm{CBI}$ (Readhead et al. 2004a), B03 (Jones et al. 2006), ACBAR (K07), and QUaD (Ade et al. 2007). The ACBAR band powers are in excellent agreement with the cosmological models constrained by observations on larger angular scales. The probability to exceed the reduced $\chi^{2}$ between the ACBAR band powers and the

\footnotetext{
14 http://cosmology.berkeley.edu/group/swlh/acbar/index.html.
}

WMAP3-only best-fit $\Lambda$ CDM model is $17 \%$. This probability increases to $62 \%$ with the WMAP5-only best-fit model. This serves as both a powerful confirmation of our basic cosmological model and an indication of the quality of the ACBAR data set.

\subsection{Anisotropies at $\ell>2000$}

Several theoretical calculations (Cooray et al. 2000; Komatsu $\&$ Seljak 2002) and hydrodynamical simulations (Bond et al. 2005; White et al. 2002) suggest that the thermal SunyaevZel'dovich effect power spectrum will exceed that of the primary CMB temperature anisotropies for $\ell \gtrsim 2500$ at $150 \mathrm{GHz}$. The amplitude of the SZE power spectrum is closely related to the amplitude of matter perturbations, which is commonly parameterized as $\sigma_{8}$; the SZE power spectrum is expected to scale as $\sigma_{8}^{7}$ (Zhang et al. 2002). To a lesser extent, the level of the SZE will also depend on details of cluster gas physics and thermal history. The nonrelativistic thermal SZE $\left(\Delta T_{\mathrm{SZ}}\right)$ has a unique frequency dependence

$$
\frac{\Delta T_{\mathrm{SZ}}}{T_{\mathrm{CMB}}}=y\left(x \frac{e^{x}+1}{e^{x}-1}-4\right),
$$

where $x=\frac{h v}{k T_{\mathrm{CMB}}}=v / 56.8 \mathrm{GHz}$. The variable $y$ is the Compton parameter and is proportional to the integrated electron pressure along the line of sight. The CBI extended mosaic observations (Readhead et al. 2004a) detected more power above $\ell=2000$ than is expected from primary CMB anisotropies. This excess could be the first detection of the SZE power spectrum (Mason et al. 2003; Readhead et al. 2004a; Bond et al. 2005). However, there are alternative explanations for the observed power ranging from an unresolved population of low-flux radio sources to nonstandard inflationary models (Cooray \& Melchiorri 2002; Griffiths et al. 2003; Subramanian et al. 2003) that produce higher than expected $\mathrm{CMB}$ anisotropy power at small angular scales. The frequency dependence of the excess power can be exploited to help discriminate between the SZE and other potential explanations.

The ACBAR band powers reported in this paper are slightly larger at $\ell>2000$ than expected for the "ACBAR+WMAP5" best-fit model. We subtract the predicted band powers at $\ell>1950$ from the measured band powers in Table 3, and find an excess of $22 \pm 20 \mu \mathrm{K}^{2}$ in a flat band power from $1950<\ell<3000$. This estimate ignores the band-power contribution from dusty protogalaxies, which is expected to be comparable (see Section 6.2). The ACBAR band powers at $150 \mathrm{GHz}$ can be used to place constraints on frequency spectrum of the larger CBI excess measured at $30 \mathrm{GHz}$. We parameterize the excess power for $\ell>1950$ at the two frequencies as $P_{30}=\alpha P_{150}$, and sample the likelihood surface for $\alpha \in[0,10]$ and $P_{150} \in[0,300] \mu \mathrm{K}^{2}$. The beam uncertainty and the calibration error for both experiments are taken into account by Monte Carlo techniques. The likelihood function is averaged over 1000 realizations under the assumption that each of the errors has a normal distribution. The resulting likelihood function for $\alpha$ (after $P_{150}$ is marginalized) is shown in Figure 7. From the ACBAR and CBI frequency bands, we expect $\alpha=4.3$ for power originating from the SZE. If the excess is due to primary CMB anisotropies, we expect $\alpha=1$. We conclude that it is more than five times as likely that the excess seen by CBI and ACBAR is caused by the thermal SZE than a primordial source. We expect the contribution of radio sources to CMB power to be at least a factor of ten higher at $30 \mathrm{GHz}$ than at $150 \mathrm{GHz}$ 


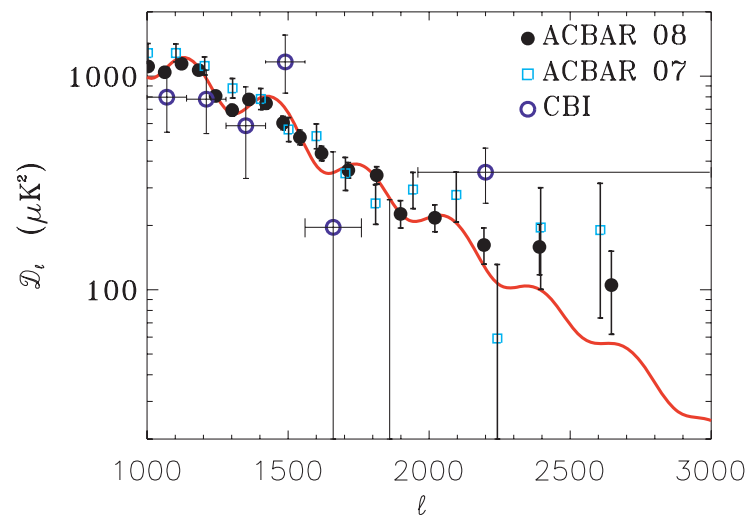

Frequency Dependence of the ACBAR and CBI Excess

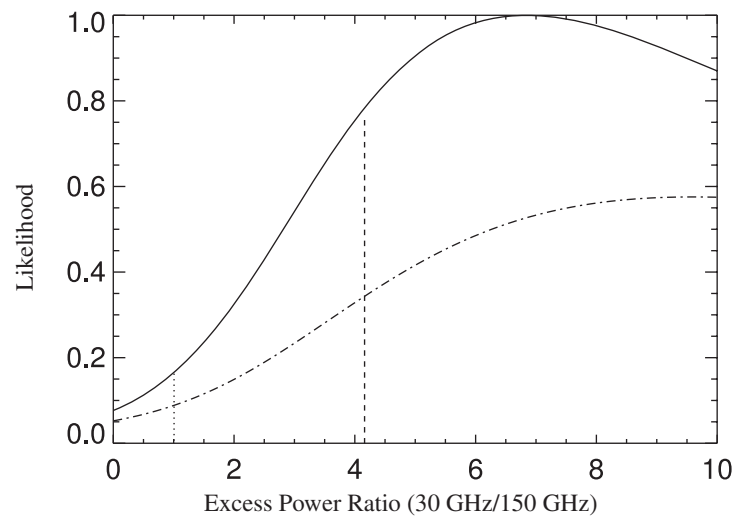

Figure 7. ACBAR results on the high $\ell$ anisotropies. Left: the ACBAR band powers above $\ell=1000$ plotted against the best-fit ACBAR+WMAP5 model spectrum. The latest CBI results at $30 \mathrm{GHz}$ and the previous ACBAR results are also shown. The ACBAR band powers for $\ell>1950$ are consistently below the reported high $\ell$ CBI band power. Right: the likelihood distribution for the ratio of the "excess" power, observed by CBI at $30 \mathrm{GHz}$ and ACBAR at $150 \mathrm{GHz}$. The solid line assumes that dusty protogalaxies do not contribute to the ACBAR band powers, while the dash-dot line includes the predicted contribution of approximately $22 \mu \mathrm{K}^{2}$ at $\ell=2600$ from these sources (see Section 6.2). With the inclusion of dusty galaxies, the likelihood peaks at a higher power ratio $(\simeq 10)$. The excess power for each experiment is the difference between the measured and model band powers for each experiment in a flat band with $\ell>1950$. The likelihood for a given power ratio is found from Monte Carlo simulations of the band powers and uncertainties. The vertical dashed line represents the expected ratio (4.3) for the excess being due to the SZE, while radio foregrounds would correspond to a ratio of $>10$. If the excess power seen in CBI is caused by nonstandard primordial processes, the ratio will be unity (black body), indicated by the dotted line. It is considerably more likely that the excess seen by CBI is caused by either the thermal SZE or radio foreground contamination than a primordial source.

$(\alpha \geqslant 10)$. Because of the relatively weak detection of excess power by ACBAR, flat spectrum radio sources are determined to be $\sim 10 \%$ more likely than the SZE to be the source of the excess. The lower level of excess power seen by ACBAR argues against the CBI excess having a primordial origin, but is consistent with either the SZE or radio source foregrounds. When we include the expected contribution of dusty protogalaxies to the ACBAR excess band power, the likelihood of the CBI excess being due to radio sources increases with respect to thermal SZE.

\section{COSMOLOGICAL PARAMETERS}

\subsection{Cosmological Parameters and their "Prior" Measures}

In this section, we estimate cosmological parameters for a minimal inflation-based, spatially flat, tilted, gravitationally lensed, $\Lambda C D M$ model characterized by six parameters, and then investigate models with additional parameters to test extensions of the theory. For our base model, the six parameters are: the physical density of baryonic and dark matter, $\Omega_{b} h^{2}$ and $\Omega_{c} h^{2}$; a uniform spectral index $n_{s}$ and amplitude $\ln A_{s}$ of the primordial power spectrum, the optical depth to last scattering, $\tau$; and $\theta$, the ratio of the sound horizon at last scattering to the angular diameter distance. The primordial comoving scalar curvature power spectrum is expressed as $\mathcal{P}_{s}(k)=A_{s}\left(k / k_{\star}\right)^{\left(n_{s}-1\right)}$, where the normalization (pivot-point) wavenumber is chosen to be $k_{\star}=0.05 \mathrm{Mpc}^{-1}$. The parameter $\theta$ maps angles observed at our location to comoving spatial scales at recombination; changing $\theta$ shifts the entire acoustic peak/valley and damping pattern of the CMB power spectra. Additional parameters are derived from this basic set. These include: the energy density of a cosmological constant in units of the critical density, $\Omega_{\Lambda}$; the age of the universe; the energy density of nonrelativistic matter, $\Omega_{m}$; the rms (linear) matter fluctuation level in $8 \mathrm{~h}^{-1} \mathrm{Mpc}$ spheres, $\sigma_{8}$; the redshift to reionization, $z_{\mathrm{re}}$; and the value of the present day Hubble constant, $H_{0}$, in units of $\mathrm{km} \mathrm{s}^{-1} \mathrm{Mpc}^{-1}$.

Single-field models of inflation predict the existence of a gravitational wave background characterized by a primordial power law $\mathcal{P}_{t} \sim k^{n_{t}}$. We characterize the strength by the tensor-to-scalar ratio $r=\mathcal{P}_{t} / \mathcal{P}_{s}$ evaluated at a pivot point $0.002 \mathrm{Mpc}^{-1}$. We relate the tilt to $r$ using the approximate consistency relation $n_{t} \approx-r / 8 /(1-r / 16)$. (We find little difference in the parameters if we just fix $n_{t}$ to be zero, as has often been assumed when $r$ is included, but using this relation is superior since it is motivated by inflation physics.)

A small running of the spectral index is also expected in slowroll inflation and we test for this by extending the basic $\Lambda \mathrm{CDM}$ power law model to include a scale dependence of the scalar spectral tilt, $d n_{s} / d \ln (k)$.

We have also added nonzero curvature $\Omega_{k}$ to our basic six parameters. The results are consistent with the flat case, but with the standard geometrical degeneracy relating $\Omega_{k}$ and $\Omega_{\Lambda}$ expressed through $\theta$ leading to a near-degenerate tail to $\Omega_{k}<0$.

The ACBAR spectrum includes band powers at $\ell>2000$ where the signal due to secondary $\mathrm{CMB}$ anisotropies associated with postrecombination nonlinear effects should become significant. In particular, the thermal Sunyaev-Zel'dovich effect and the contribution of unresolved radio sources and dusty galaxies will ultimately dominate over the primary anisotropy damping tail; the only question is at what multipole crossover occurs. Without including such secondary effects, the parameters we derive from the primary anisotropy power spectrum could be biased. To account for this, we have added to the primary anisotropy power spectrum (1) the SZE template power spectrum $\hat{\mathcal{D}}_{\ell}^{\text {SZ }}$ used in K07 and (Bond et al. 2005; Goldstein et al. 2003), which was derived from cosmological hydrodynamics simulations, and (2) an unclustered point source template as in Equation (3). Each template is scaled by an overall amplitude parameter, $q_{\mathrm{sz}}$ and $q_{\mathrm{src}}$, which we assume have uniform prior measures with a range much larger than required by the ACBAR data. The white-noise form for $\mathcal{D}_{\ell}^{\text {src }}$ given by Equation (3) is appropriate for the statistically averaged power of a distribution of unclustered sources. The clustering of radio sources is not a large effect, but we do expect submm sources associated with 
dusty galaxies at lower flux levels to be clustered. As mentioned in Section 6.2, in spite of great strides in submm observations in recent years, significant uncertainties remain in source fluxes and clustering at $150 \mathrm{GHz}$. Theoretical models suggest both will be important for a complete treatment, but the approximation adopted here should be sufficient for the ACBAR data set. In the parameter tables below, we show results including these secondary templates. We find the basic parameter central values and uncertainties change little whether we marginalize over either of the two template amplitudes or set them to zero.

The parameter constraints are obtained using a Monte Carlo Markov Chain (MCMC) sampling of the multidimensional likelihood as a function of model parameters. The pipeline is based on the publicly available CosmoMC ${ }^{15}$ package (Lewis $\&$ Bridle 2002). CMB angular power spectra and matter power spectra are computed using the CAMB code (Lewis et al. 2000). As described in Section 7, we approximate the full non-Gaussian band-power likelihoods with an offset lognormal distribution (Bond et al. 2000). Our standard CosmoMC results include the effects of weak gravitational lensing on the CMB (Seljak 1996; Lewis et al. 2000). Lensing effects in the temperature spectrum are expected to become significant at scales $\ell>1000$, hence it is important to include this effect when interpreting the ACBAR results. The major effect of lensing is a scale-dependent smoothing of the angular power spectrum that diminishes the peaks and valleys of the spectrum. Inclusion of lensing in the model improves the fit to the data for all experiment combinations.

The typical computation consists of eight separate chains, each having different initial random parameter choices. The chains are run until the largest eigenvalue of the Gelman-Rubin test is smaller than 0.01 after accounting for burn-in. Uniform priors with very broad distributions are assumed for the basic parameters. The standard run also includes a weak prior on the Hubble constant $\left(45<H_{0}<90 \mathrm{~km} \mathrm{~s}^{-1} \mathrm{Mpc}^{-1}\right)$ and on the age of the universe ( $>10 \mathrm{Gyr}$ ), but these have negligible effects. We also investigate the influence of adding large-scale structure (LSS) data from the 2 degree Field Galaxy Redshift Survey (2dFGRS) (Cole et al. 2005) and the Sloan Digital Sky Survey (SDSS) (Tegmark et al. 2006). When including the LSS data, we use only the band powers for length scales larger than $k \sim 0.1 \mathrm{~h} \mathrm{Mpc}^{-1}$ to avoid nonlinear clustering and scale-dependent galaxy biasing effects. We marginalize over a parameter $b_{g}^{2}$ that describes the (linear) biasing of the galaxygalaxy power spectrum for $L_{\star}$ galaxies relative to the underlying mass density power spectrum. We adopt a Gaussian prior on $b_{g}^{2}$ centered around $b_{g}=1.0$ with a very large width. We have also tried restricting the width to $\delta b_{g}=0.3$, but the cosmic parameters are insensitive to this width.

\subsection{Base Parameter Results}

The results for the basic spatially flat tilted $\Lambda \mathrm{CDM}$ parameters are presented in Table 4 . The confidence limits are obtained by marginalizing the multidimensional likelihoods down to one dimension. The median value is obtained by finding the $50 \%$ integral of the resulting likelihood function while the lower and upper error limits are obtained by finding the $16 \%$ and $84 \%$ integrals, respectively. The CMBall data combination includes the ACBAR results presented here and other CMB data sets with published band powers and window functions: the WMAP 5-year angular power spectra (Nolta et al. 2008),

\footnotetext{
15 http://cosmologist.info/cosmomc.
}

and for comparison the WMAP 3-year spectra (Hinshaw et al. 2006); the CBI extended mosaic results (Readhead et al. 2004a) and polarization results (Readhead et al. 2004b; Sievers et al. 2005) combined in the manner described in Sievers et al. (2005); ${ }^{16}$ the DASI two-year results (Halverson et al. 2002); the DASI EE and TE band powers (Leitch et al. 2005); the VSA final results (Dickinson et al. 2004); the MAXIMA 1998 flight results (Hanany et al. 2000); and the TT, TE, and EE results from the BOOMERANG 2003 flight (Jones et al. 2006; Piacentini et al. 2006; Montroy et al. 2006). Only $\ell>350$ band powers are included for BOOMERANG because of overlap with WMAP (although inclusion of the lower $\ell$ results leaves the parameter results essentially unchanged). While ACBAR and BOOMERANG are both calibrated through WMAP, this is a small contribution to the total uncertainty in the ACBAR calibration and we treat the calibration uncertainties as independent in our parameter analysis. Although the DASI, CBI, and BOOMERANG $2003 \mathrm{EE}$ and TE results for high $\ell$ polarization are included, they have little impact on the values of the parameters we obtain.

The latest WMAP likelihood code found at http://lambda.gsfc. nasa.gov/ has been used in our analyses. When this ACBAR paper was submitted, all parameter analyses were done using WMAP3 (Hinshaw et al. 2006); the new WMAP5 results came out a few months later. The WMAP5 data allowed for an improved cross-calibration of the ACBAR and WMAP band powers, and resolved the $(<1-\sigma)$ parameter tensions that existed between the WMAP3 and ACBAR results. We note that the WMAP5 team's parameter analysis (Dunkley et al. 2008) made use of the ACBAR band powers with the $1.4 \%$ higher temperature calibration from the WMAP 3 and ACBAR crosscalibration. The marginalized one-dimensional likelihood distributions for the basic parameter set we obtain are shown in Figure 8. Note the contrast between the parameter determinations for WMAP3 and WMAP5. The primary improvement of WMAP5 over WMAP3 was a better understanding of the beam, which resulted in an improved measurement of the third acoustic peak. Other improvements in WMAP5 were updated point source correction, stimulated by (Huffenberger et al. 2006), and foreground marginalization on large angular scales.

The addition of LSS data has little impact on the mean values and errors in the cosmic parameters. The largest shift is $<1-\sigma$ in $\Omega_{c} h^{2}$, from $0.111_{-0.005}^{+0.005}$ to $0.107_{-0.004}^{+0.004}$. Our LSS results only include information on the shape of the density power spectrum, not its overall amplitude since we marginalize over the galaxy bias factor. If results from weak lensing are included in the LSS data, then there is a slight increase in $\sigma_{8}$, but it depends somewhat on which lensing results are included. The effect is to slightly increase the CMB+LSS result and improve the consistency with the CMBall results. The third peak is well determined by both ACBAR and WMAP5, and this defines the dark matter density; the inclusion of the LSS data does not significantly improve the constraints. We have also found that the results do not significantly change from CMBall+LSS when SN1a data are included (in this case, from the Riess et al. (2004) gold set), so we have not included a separate column. Including SN1a data would be crucial if we were attempting to constrain the equation of state of dark energy.

\footnotetext{
16 We exclude the band powers below $\ell=600$ from the CBI extended mosaic results to reduce the correlation with the TT band powers of the CBI polarization data set, which influence the sample-dominated end of the spectrum.
} 

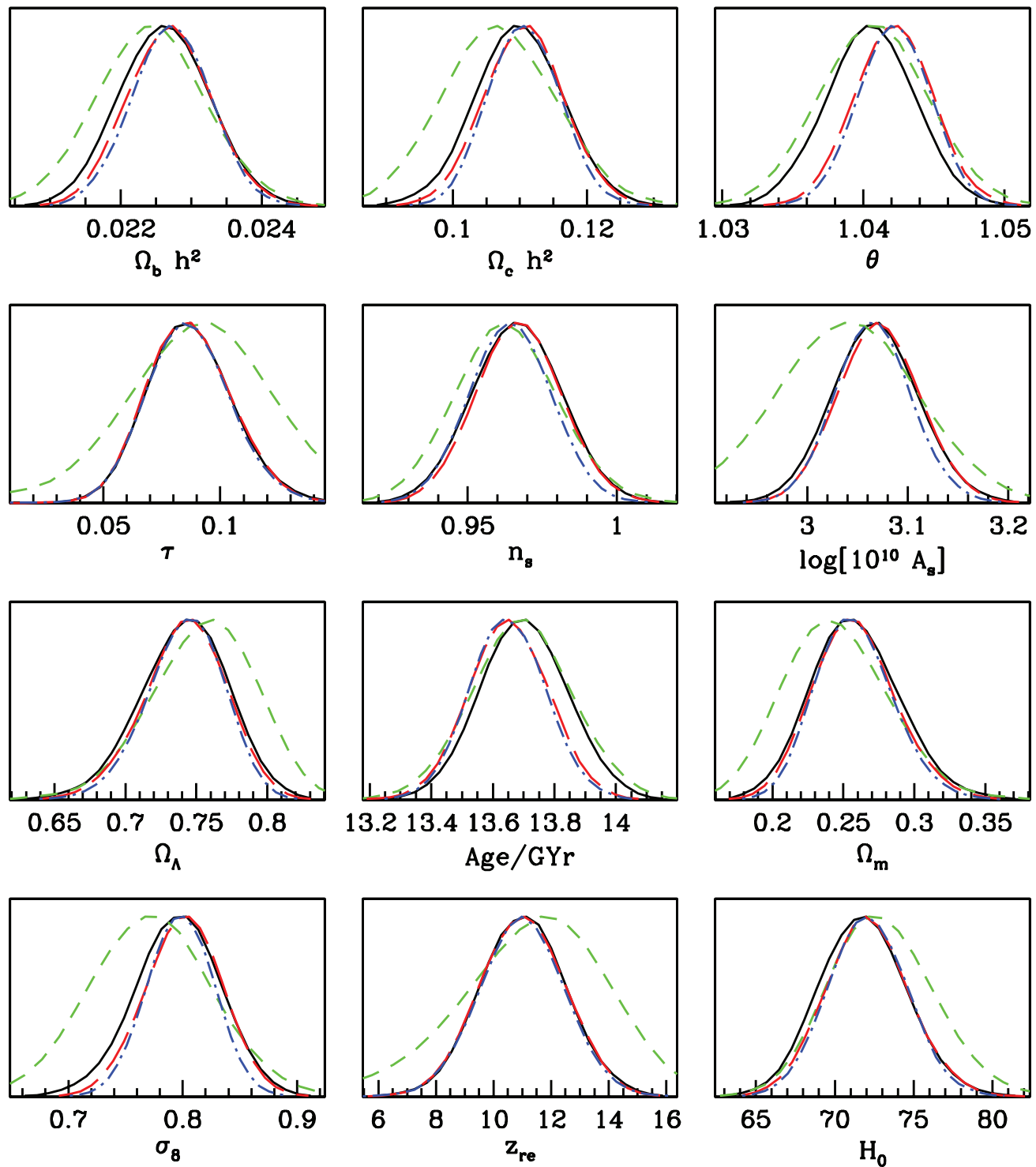

Figure 8. Basic parameter marginalized one-dimensional likelihood distributions for the following data combinations: WMAP3-only (green, dashed), WMAP5-only (black, solid), ACBAR+WMAP5 (red, long-dashed), and CMBall (blue, dot-dashed). All runs include lensing.

All parameter results listed in Tables 4 and 5 include the effects of weak lensing of the CMB on the resulting power spectrum. For every case including CMB lensing, we have performed an identical calculation, neglecting the effects of lensing. Including lensing improves the fit of the model to the observed band powers compared for all data combinations. This can be quantified by the log-ratio of the lensed to no-lensed Bayesian evidence, $\Delta \ln \mathcal{E}=\ln [P($ lens $\mid$ data, theory $) / \mathrm{P}$ (nolens $\mid$ data, theory)]. The evidence $P$ (lens $\mid$ data, theory) is an integral of the product of the a priori probability (the parameters' measure) and the likelihood of data given those parameters; it appears in the denominator in the Bayesian chain to ensure the a posteriori probability has unity normalization. The resulting number is a conditional probability given the data and the assumptions about the parameters. The parameters and their measures are exactly the same, so the ratio is a robust indicator of preference. For WMAP5 alone it is $\Delta \ln \mathcal{E}=2.04$; it increases to 2.89 with ACBAR included; and is 2.63 for CMBall. Naively relating this to a Gaussian translates to a significance of $\sim 2.3 \sigma$ for CMBall. From Figure 5, it is clear that the difference in the power spectra of lensing and no-lensing is small; for this plot, the best-fit parameters from the lensed analysis were fixed and used to compute a lensed and unlensed spectrum. The difference, $\Delta C_{\ell}^{\text {lens }} \equiv C_{\ell}^{\text {lens }}-C_{\ell}^{\text {no-lens }}$, is explicitly shown in the inset of Figure 10. We find only small shifts in the median value of the cosmic parameters when lensing is included; e.g., for the ACBAR+WMAP5 data combination, we find $\sigma_{8}=0.79_{-0.03}^{+0.03} \rightarrow 0.80_{-0.03}^{+0.03}$ and $\Omega_{c} h^{2}=0.109_{-0.006}^{+0.006} \rightarrow$ $0.111_{-0.006}^{+0.006}$ when going from nonlensed to lensed models, respectively.

We now test whether the strength of the lensing modification is consistent with expectations for lensing, by multiplying the lensing template $\Delta C_{\ell}^{\text {lens }}$, which varies with cosmic parameters, by a variable strength $q_{\text {lens }}$

$$
C_{\ell}^{\text {lens }}=C_{\ell}^{\text {no-lens }}+q_{\text {lens }} \Delta C_{\ell}^{\text {lens }} .
$$

The normalization is such that $q_{\text {lens }}=1$ gives the normal 


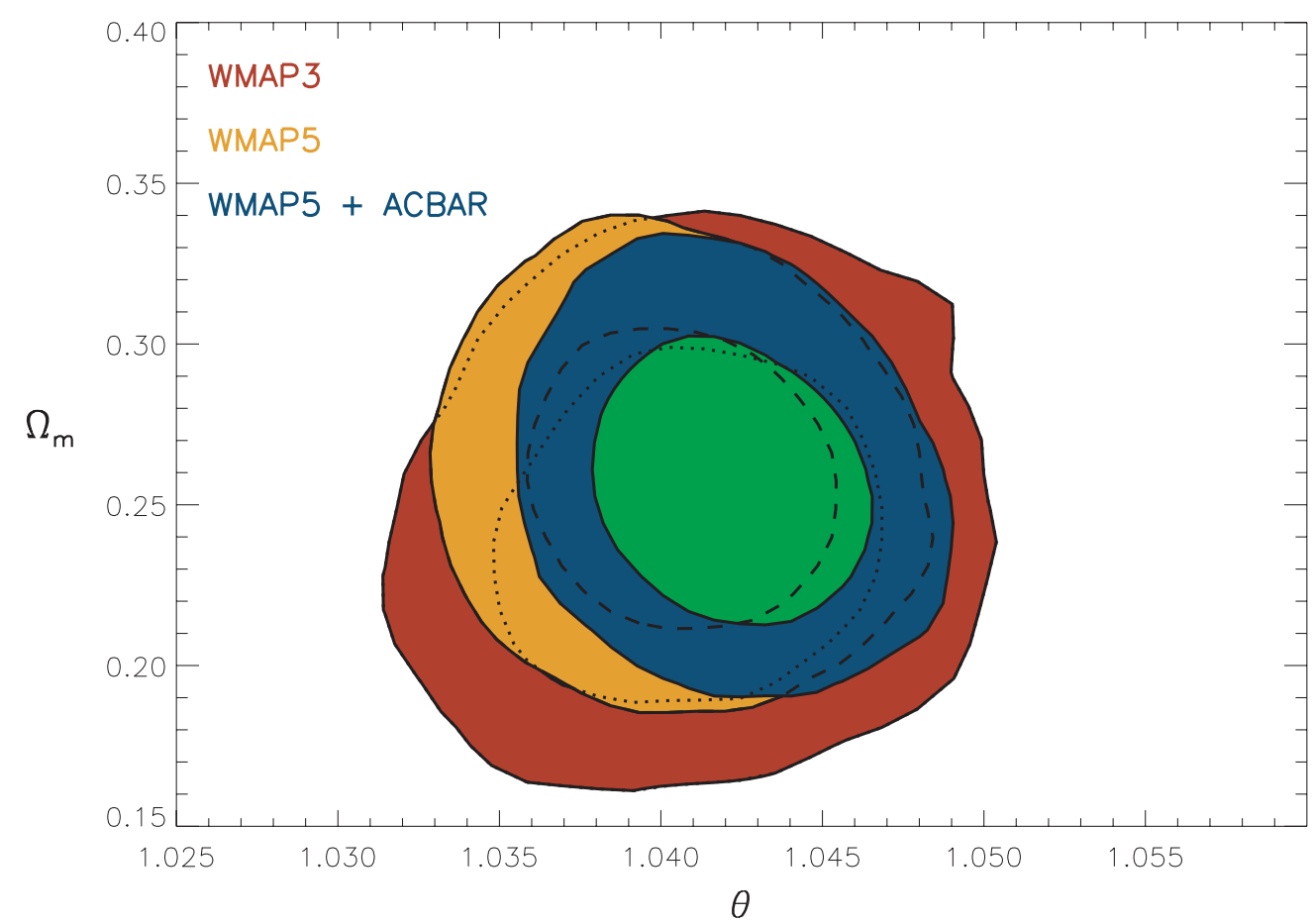

Figure 9. 68\% and 95\% 2D marginalized contours in $\theta$ and $\Omega_{m}$ for a number of data combinations. The results for $\theta$ from WMAP are largely driven by the determination of the third peak. The value preferred by the WMAP5 data is slightly higher than that from WMAP3, and is more consistent with that found when ACBAR is added to either WMAP data set.

Table 4

Basic Six Parameter Constraints

\begin{tabular}{|c|c|c|c|c|c|c|}
\hline & WMAP5 & WMAP5+ACBAR & CMBall & CMBall+LSS & $\mathrm{CMBall}+q_{\mathrm{SZ}}$ & $\mathrm{CMBall}+\mathrm{SZ}+q_{\mathrm{src}}$ \\
\hline$\Omega_{b} h^{2}$ & $0.0226_{-0.0006}^{+0.0006}$ & $0.0227_{-0.0006}^{+0.0006}$ & $0.0227_{-0.0005}^{+0.0005}$ & $0.0228_{-0.0005}^{+0.0005}$ & $0.0227_{-0.0005}^{+0.0006}$ & $0.0227_{-0.0005}^{+0.0005}$ \\
\hline$\Omega_{c} h^{2}$ & $0.110_{-0.006}^{+0.006}$ & $0.111_{-0.006}^{+0.006}$ & $0.111_{-0.005}^{+0.005}$ & $0.107_{-0.004}^{+0.004}$ & $0.109_{-0.005}^{+0.005}$ & $0.111_{-0.005}^{+0.005}$ \\
\hline$\theta$ & $1.041_{-0.003}^{+0.003}$ & $1.042_{-0.003}^{+0.003}$ & $1.042_{-0.002}^{+0.002}$ & $1.042_{-0.002}^{+0.002}$ & $1.042_{-0.002}^{+0.002}$ & $1.042_{-0.002}^{+0.002}$ \\
\hline$\tau$ & $0.086_{-0.008}^{+0.008}$ & $0.086_{-0.008}^{+0.008}$ & $\begin{array}{l}0.086_{-0.008}^{+0.0002} \\
0.008\end{array}$ & $\begin{array}{l}0.087_{-0.008}^{+0.002} \\
0.008\end{array}$ & $\begin{array}{l}0.086_{-0.008}^{+0.008} \\
0.008\end{array}$ & $\begin{array}{l}0.085_{-0.008}^{+0.002} \\
0.008\end{array}$ \\
\hline$n_{s}$ & $0.967_{-0.015}^{+0.015}$ & $0.967_{-0.014}^{+0.014}$ & $0.964_{-0.013}^{+0.013}$ & $0.968_{-0.012}^{+0.012}$ & $0.962_{-0.013}^{+0.013}$ & $0.962_{-0.013}^{+0.013}$ \\
\hline $\mathcal{A}_{s}$ & $3.07_{-0.04}^{+0.04}$ & $3.07_{-0.04}^{+0.04}$ & $3.07_{-0.04}^{+0.04}$ & $3.05_{-0.04}^{+0.04}$ & $3.05_{-0.04}^{+0.04}$ & $3.06_{-0.03}^{+0.04}$ \\
\hline$\Omega_{\Lambda}$ & $0.74_{-0.03}^{+0.03}$ & $0.74_{-0.03}^{+0.03}$ & $0.74_{-0.03}^{+0.04}$ & $0.76_{-0.02}^{+0.04}$ & $0.75_{-0.03}^{+0.02}$ & $0.74_{-0.03}^{+0.02}$ \\
\hline Age & $13.7_{-0.1}^{+0.1}$ & $13.7_{-0.1}^{+0.1}$ & $13.6_{-0.1}^{+0.1}$ & $13.6_{-0.1}^{+0.1}$ & $13.7_{-0.1}^{+0.1}$ & $13.7_{-0.1}^{+0.1}$ \\
\hline$\Omega_{m}$ & $0.26_{-0.03}^{+0.03}$ & $0.26_{-0.03}^{+0.03}$ & $0.26_{-0.02}^{+0.03}$ & $0.24_{-0.02}^{+0.02}$ & $0.25_{-0.02}^{+0.03}$ & $0.26_{-0.02}^{+0.03}$ \\
\hline$\sigma_{8}$ & $0.80_{-0.04}^{+0.04}$ & $0.80_{-0.03}^{+0.03}$ & $0.80_{-0.03}^{+0.03}$ & $0.78_{-0.02}^{+0.03}$ & $0.79_{-0.03}^{+0.03}$ & $0.80_{-0.03}^{+0.03}$ \\
\hline$z_{\text {re }}$ & $11.0_{-1.4}^{+1.4}$ & $11.0_{-1.4}^{+1.0 .05}$ & $11.0_{-1.4}^{+1.0 .03}$ & $11.0_{-1.4}^{+1.0 .02}$ & $10.9_{-1.4}^{+1.4}$ & $10.9^{+1.4}$ \\
\hline$H_{0}$ & $71.8_{-2.7}^{+2.7^{4}}$ & $72.0_{-2.5}^{+2.6^{+}}$ & $72.1_{-2.4}^{+2.4}$ & $73.6_{-1.9}^{+1.9^{4}}$ & $72.5_{-2.4}^{+2.4}$ & $72.0_{-2.3}^{+2.4}$ \\
\hline$q_{\mathrm{SZ}}$ & $\ldots$ & $\ldots$ & $\ldots$ & $\ldots$ & $0.69_{-0.11}^{+0.11}$ & $\ldots$ \\
\hline$q_{\mathrm{src}}$ & $\cdots$ & $\ldots$ & $\ldots$ & $\ldots$ & $\ldots$ & $29_{-28}^{+12}$ \\
\hline$\sigma_{8}^{\mathrm{SZ}}$ & $\ldots$ & $\ldots$ & $\ldots$ & $\ldots$ & $0.93_{-0.05}^{+0.04}$ & $\ldots$ \\
\hline
\end{tabular}

Notes. Results for the basic parameter set. All runs assume flat cosmologies, uniform, and broad priors on each of the basic six parameters, and a weak prior on the Hubble constant $\left(45<H_{0}<90 \mathrm{~km} \mathrm{~s}^{-1} \mathrm{Mpc}^{-1}\right)$ and the age of the universe ( $>10 \mathrm{Gyr}$ ). Here $\mathcal{A}_{s} \equiv \log \left[10^{10} A_{s}\right]$. All runs include the effect of weak gravitational lensing on the CMB. Column 5 presents the results when an $\mathrm{SZ}$ template characterized by the overall SZ template-power $q_{\mathrm{SZ}}$ is included. The values of $\sigma_{8}^{\mathrm{SZ}}=q_{\mathrm{SZ}}^{1 / 7}\left(\Omega_{b} h\right)^{2 / 7}$ are higher than the $\sigma_{8}$ derived from the primary anisotropies. Column 6 shows the results when the point source contribution scaled by $q_{\text {src }}$ is included in combination with the SZ template with $q_{\mathrm{SZ}}$ set to $\sigma_{8}^{7}\left(\Omega_{b} h\right)^{2}$. The marginalization over either extra high $\ell$ contribution does not significantly shift the results for the basic parameters.

lensed CMB spectrum, while $q_{\text {lens }}=0$ gives the no-lensing case. An accurate determination of this subtle effect requires highly accurate window functions for the bands. We use a flat prior probability for $q_{\text {lens }}$, allowing it to vary from 0 to 10. With WMAP5 alone, we obtain $q_{\text {lens }}=1.34_{-0.26(-0.85)}^{+0.27(1.51)}$;
WMAP5+ACBAR gives $q_{\text {lens }}=1.23_{-0.23(-0.76)}^{+0.21(+0.83)}$; CMBall gives $q_{\text {lens }}=1.21_{-0.24(-0.76)}^{+0.24(0.82)}$. We have also listed the two-sigma errors (in parentheses), which are far from twice the one-sigma values, reflecting the highly non-Gaussian nature of the marginalized likelihoods evident in the figure. Although we emphasize that 


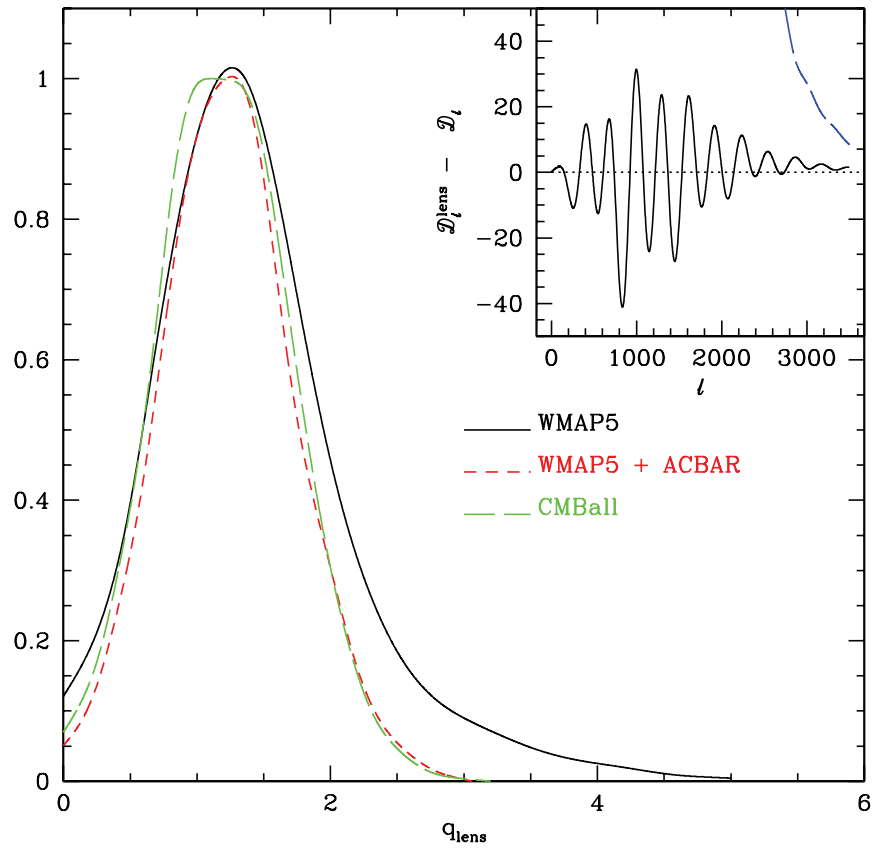

Figure 10. One-dimensional marginalized likelihood distribution for the lensing amplitude $q_{\text {lens }}$ for the combinations of CMB data shown. Although only $q_{\text {lens }}=1$ is physically meaningful, these distributions indicate that the data prefer lensing of about the right magnitude. The ratio of the value at $q_{\text {lens }}=1$ to that at $q_{\text {lens }}=0$ is the Bayesian evidence that lensing at the right amplitude is preferred over no-lensing. The inset figure shows $\Delta \mathcal{D}_{\ell}^{\text {lens }} \equiv \mathcal{D}_{\ell}^{\text {lens }}-\mathcal{D}_{\ell}^{\text {no-lens }}$, with both spectra computed using the best-fit parameters from the lensed analysis of the WMAP5+ACBAR data. Notice how small this correction is relative to $\mathcal{D}_{\ell}^{\text {lens }}$, shown as the dashed blue curve. The cosmological parameters used to construct $\Delta \mathcal{D}_{\ell}^{\text {lens }}$ have been allowed to vary as the data require in constructing the marginalized likelihoods for $q_{\text {lens }}$. Each parameter variation leads to a slightly different difference template than that shown in the inset.

$q_{\text {lens }}$ is not in any sense an independent parameter, it does illustrate that lensing of the expected strength is preferred. ${ }^{17}$ The significance of the detection is somewhat less than the WMAP3/NVSS/SDSS cross-correlation results of Smith et al. (2007) and Hirata et al. (2008). In Figure 10, we show the marginalized distribution for the $q_{\text {lens }}$ parameter using various combinations of CMB data.

We have also run a limited set of nonflat model chains. The models in this case do not include the effect of weak lensing and we keep the same weak prior on $H_{0}$. When $\Omega_{k}=0$ is not enforced, the weak prior on $H_{0}$ has a significant effect on the result as it restricts the extent of the geometrical degeneracy, which is present in this case. For WMAP5-only, we obtain $\Omega_{k}=-0.018_{-0.026}^{+0.027}$, which becomes $\Omega_{k}=-0.013_{-0.029}^{+0.019}$ when ACBAR is included.

\subsection{Residual Source Marginalization}

As discussed in Section 6.2, the ACBAR band powers at $\ell>1950$ marginally exceed the predictions of the best-fit models for the primary $\mathrm{CMB}$. We have repeated the basic parameter runs including an unclustered source contribution

\footnotetext{
17 Calabrese et al. (2008) also undertook a lensing analysis of ACBAR temperature power spectrum, but, instead of $q_{\text {lens }}$ as defined here, they used a multiplier $A_{\mathrm{L}}$ of the lensing potential power spectrum, defined to be unity for normal lensing; they found $A_{\mathrm{L}}=3.0_{-0.9}^{+0.9}$ for WMAP5+ACBAR. Repeating our analysis with this parameterization, we find lower values,

$A_{\mathrm{L}}=1.60_{-0.26(-0.99)}^{+0.55(+1.79)}$. With the highest three ACBAR bins cut out, where secondary effects might have an impact on the determination of the lensing strength parameter, the results are essentially unchanged.
}

described by Equation (3) and marginalize over a wide uniform prior in $q_{\text {src }}$ from 0 to $4600 \mu \mathrm{K}^{2}$, i.e., more than 100 times the power required to fit the high $\ell$ points. The results for runs including this marginalization are shown in Tables 4 and 5. The template amplitude $q_{\text {src }}$ is not well constrained by the fit, and the effect of the marginalization on the basic parameters is negligible. The addition of a source template improves the fit to the high $\ell$ points, and therefore increases, albeit modestly, the best-fit likelihood: for the ACBAR+WMAP5 combination the change in likelihood is $\Delta \ln L=0.24$. The value and uncertainty for $q_{\text {src }}$ given in Table 4 spans the range of predictions for the contribution from dusty protogalaxies.

Contributions from point sources or the SZE are nearly degenerate in the high $\ell$ ACBAR band powers. However, the CMBall combination is potentially sensitive to an SZE contribution because of the SZE frequency dependence. The CBI data have a more significant excess at high $\ell$; however, through substantial modeling and vetoing, the CBI band powers are expected to be free of radio source contamination. We assume that the residual contribution of the unresolved source background to the CBI band powers is sufficiently low that we do not require a source template for that data, only the SZE template.

\subsection{Sunyaev-Zel'dovich Template Extension}

The amplitude of the SZE signal depends strongly on the overall matter fluctuation amplitude, $\sigma_{8}$. We have modified our parameter fitting pipeline to allow for extra frequency dependent contributions to the CMB power spectrum and have implemented it in a simple analysis using a fixed template $\hat{\mathcal{D}}_{\ell}^{\mathrm{SZ}}$ for the shape of the thermal SZE power spectrum. The template was obtained from two large, hydrodynamical simulations of a scale-invariant $\left(n_{s}=1\right) \Lambda \mathrm{CDM}$ model with $\Omega_{b} h=0.029$ and with $\sigma_{8}=0.9$ and $\sigma_{8}=1.0$ (see Bond et al. 2005, for a detailed description of the simulations). Recently, the WMAP team have used a different SZE template based on analytic estimations of the power spectrum (Spergel et al. 2006). It is characterized by a slower rise in $\ell$ than the simulation-based template, which cut nearby clusters out of the power spectrum. There has been no fine-tuning of either spectra to agree with all of the X-ray and other cluster data. This may have an effect on shape, especially at high $\ell$.

The SZE contribution, $\mathcal{D}_{\ell}^{\mathrm{SZ}}=\left(q_{\mathrm{SZ}}\right) f_{\nu} \hat{\mathcal{D}}_{\ell}^{\mathrm{SZ}}$, added to the base six-parameter model spectrum has a frequency-dependent SZE prefactor $f_{v}$. Including this SZE template with all model parameters free to vary is complementary to the analysis of Section 7.2, which directly compared the residual CBI and ACBAR band powers at $\ell>1950$ for the best fit WMAP5+ACBAR model power spectrum. In that more restrictive analysis, the primary power spectrum is fixed and $f_{v}$ is allowed to vary as well as a broadband excess power. We found the ratio of excess power seen by CBI and ACBAR to be compatible with the ratio of the frequency prefactors $f_{v}$ at $30 \mathrm{GHz}$ and $150 \mathrm{GHz}$ for the Sunyaev-Zel'dovich effect, although foreground contamination of both experiments could also contribute to the observed excess. In this section, we assume that all the excess power seen by the CBI experiment is due to the SZE. In the hydrodynamical simulations used to derive the SZE template, the amplitude was shown to scale as $q_{\mathrm{SZ}}=\left(\sigma_{8} / 0.9\right)^{7}\left(\Omega_{b} h / 0.029\right)^{2}$. We consider two cases: (1) the scaling parameter $q_{\mathrm{SZ}}$ is slaved to the results from the hydrodynamical simulations, which means that it is primarily determined by the primary $\mathrm{CMB}$ data; (2) $q_{\mathrm{SZ}}$ is allowed to float freely and an independent $\sigma_{8}^{\mathrm{SZ}}$ is derived, to be 


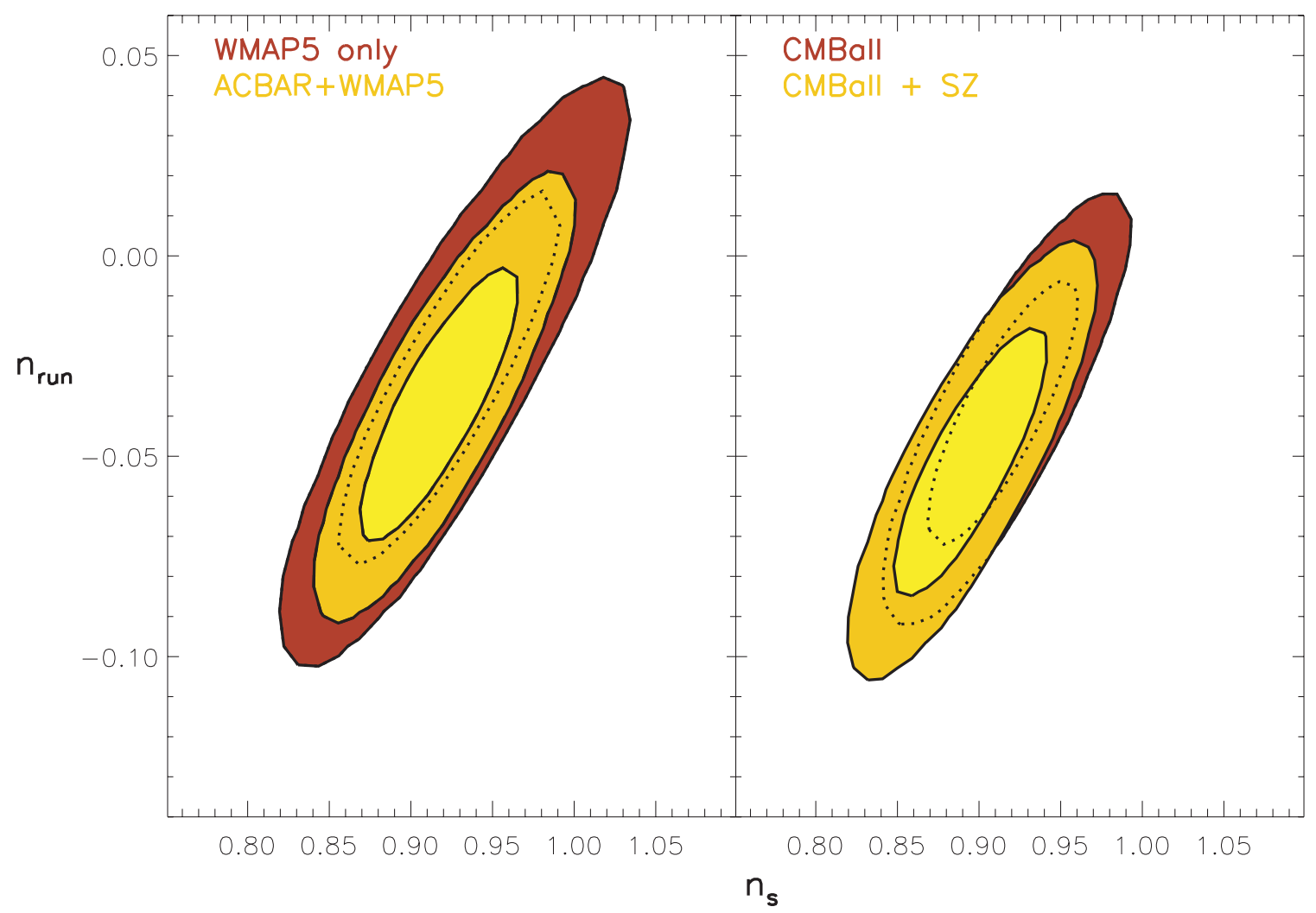

Figure 11. 68\% and 95\% 2D marginalized contours in $n_{s}$ and $n_{\mathrm{run}}=d n_{s} / d \ln k$. The left panel shows the results for the WMAP5 and ACBAR+WMAP5 combinations. The right panel shows the effect of adding the rest of the CMB data and marginalizing over the SZE template. The basic parameters are essentially unchanged if we marginalize over one or the other of the two template amplitudes.

Table 5

Running Spectral Index Parameter Constraints

\begin{tabular}{|c|c|c|c|c|c|c|}
\hline & WMAP5 & WMAP5+ACBAR & CMBall & CMBall+LSS & $\mathrm{CMBall}+q_{\mathrm{SZ}}$ & $\mathrm{CMBall}+\mathrm{SZ}+q_{\mathrm{src}}$ \\
\hline$\overline{\Omega_{b} h^{2}}$ & $0.0219_{-0.0008}^{+0.0009}$ & $0.0219_{-0.0007}^{+0.0007}$ & $0.0220_{-0.0006}^{+0.0006}$ & $0.0225_{-0.0006}^{+0.0006}$ & $0.0217_{-0.0006}^{+0.0006}$ & $0.0217_{-0.0006}^{+0.0007}$ \\
\hline$\Omega_{c} h^{2}$ & $0.117_{-0.009}^{+0.009}$ & $0.119_{-0.008}^{+0.008}$ & $0.120_{-0.008}^{+0.008}$ & $0.109_{-0.005}^{+0.005}$ & $0.122_{-0.008}^{+0.008}$ & $0.124_{-0.008}^{+0.008}$ \\
\hline$\theta$ & $1.040_{-0.003}^{+0.003}$ & $1.041_{-0.003}^{+0.003}$ & $1.042_{-0.002}^{+0.002}$ & $1.042_{-0.002}^{+0.002}$ & $1.041_{-0.002}^{+0.003}$ & $1.041_{-0.002}^{+0.002}$ \\
\hline$\tau$ & $0.090_{-0.008}^{+0.008}$ & $0.091_{-0.008}^{+0.009}$ & $0.092_{-0.008}^{+0.009}$ & $0.091_{-0.008}^{+0.009}$ & $0.092_{-0.009}^{+0.009}$ & $0.094_{-0.009}^{+0.009}$ \\
\hline$n_{s}$ & $0.923_{-0.041}^{+0.043}$ & $0.918_{-0.031}^{+0.0033}$ & $0.914_{-0.029}^{+0.0038}$ & $0.948_{-0.025}^{+0.026}$ & $0.894_{-0.029}^{+0.0031}$ & $0.896_{-0.030}^{+0.030}$ \\
\hline$d n_{s} / d \ln (k)$ & $-0.031_{-0.028}^{+0.041}$ & $-0.037_{-0.022}^{+0.023}$ & $-0.039_{-0.021}^{+0.021}$ & $-0.016_{-0.018}^{+0.019}$ & $-0.052_{-0.021}^{+0.022}$ & $-0.052_{-0.022}^{+0.022}$ \\
\hline $\mathcal{A}_{s}$ & $3.09_{-0.05}^{+0.05}$ & $3.10_{-0.04}^{+0.05}$ & $3.10_{-0.04}^{+0.05}$ & $3.07_{-0.04}^{+0.04}$ & $3.10_{-0.04}^{+0.05}$ & $3.11_{-0.04}^{+0.05}$ \\
\hline$\Omega_{\Lambda}$ & $0.70_{-0.06}^{+0.05}$ & $0.69_{-0.05}^{+0.04}$ & $0.69_{-0.05}^{+0.04}$ & $0.75_{-0.03}^{+0.02}$ & $0.68_{-0.05}^{+0.04}$ & $0.67_{-0.05}^{+0.04}$ \\
\hline Age & $13.8_{-0.2}^{+0.2}$ & $13.8_{-0.1}^{+0.1}$ & $13.8_{-0.1}^{+0.1}$ & $13.7_{-0.1}^{+0.1}$ & $13.8_{-0.1}^{+0.1}$ & $13.8_{-0.1}^{+0.1}$ \\
\hline$\Omega_{m}$ & $0.30_{-0.05}^{+0.06}$ & $0.31_{-0.04}^{+0.05}$ & $0.31_{-0.04}^{+0.05}$ & $0.25_{-0.02}^{+0.03}$ & $0.32_{-0.04}^{+0.05}$ & $0.33_{-0.04}^{+0.05}$ \\
\hline$\sigma_{8}$ & $0.81_{-0.04}^{+0.04}$ & $0.83_{-0.03}^{+0.03}$ & $0.83_{-0.03}^{+0.03}$ & $0.79_{-0.03}^{-0.02}$ & $0.83_{-0.03}^{+0.03}$ & $0.84_{-0.03}^{-0.03}$ \\
\hline$z_{\mathrm{re}}$ & $11.7_{-1.7}^{+1.04}$ & $\begin{array}{l}-0.03 \\
11.9_{-1.6}^{+1.7}\end{array}$ & $12.0_{-1.6}^{+1.03}$ & $11.5_{-1.6}^{+1.03}$ & $12.2_{-1.7}^{+1.03}$ & $12.4_{-1.7}^{+1.83}$ \\
\hline$H_{0}$ & $68.4_{-3.8}^{+4.2}$ & $67.9_{-3.3}^{+1.5}$ & $\begin{array}{r}67.7_{-3.2}^{+3.3} \\
3_{-3}\end{array}$ & $72.4_{-2.4}^{+1.4}$ & $66.7_{-3.1}^{+3.1}$ & $66.2_{-3.2}^{+3.1}$ \\
\hline$q_{\mathrm{SZ}}$ & $\cdots$ & $\cdots$ & $\cdots$ & $\cdots$ & $0.77_{-0.11}^{+0.1 \frac{1}{2}}$ & \\
\hline$q_{\mathrm{src}}$ & $\ldots$ & $\ldots$ & $\ldots$ & $\cdots$ & $\ldots$ & $30_{-29}^{+11}$ \\
\hline$\sigma_{8}^{q \mathrm{SZ}}$ & $\cdots$ & $\cdots$ & $\cdots$ & $\cdots$ & $0.93_{-0.05}^{+0.04}$ & -29 \\
\hline
\end{tabular}

Notes. Marginalized parameter constraints for models with a running of the spectral index. The tendency for the low $\ell$ data to prefer negative values continues with the higher $\ell$ data, but at less than two sigma for CMB only. The last two columns include marginalization over extra high $\ell$ contributions from SZ and point sources. The basic parameters are stable with respect to this marginalization, while the median value for $d n_{s} / d \ln k\left(k_{\star}\right)$ is increased slightly when the $\mathrm{SZ}$ or point source contribution is included.

compared with the $\sigma_{8}$ that is derived from the basic six parameters. We use a uniform prior in $q_{\mathrm{SZ}}$ in this case with limits $0 \leqslant q_{\mathrm{SZ}} \leqslant 4.0$.

Regardless of the data combination, we find that including an SZE component in the model has little effect on the values of most basic cosmological parameters (see Table 4), whether $q_{\mathrm{SZ}}$ is related to cosmic parameters through $q_{\mathrm{SZ}}=$ $\left(\sigma_{8} / 0.9\right)^{7}\left(\Omega_{b} h / 0.029\right)^{2}$ or is allowed to float freely. Note that the SZE results break the $A_{s} e^{-2 \tau}$ near-degeneracy (as does weak lensing, though not as strongly).

With the combination of the ACBAR and WMAP5 data, for which there is only a weak indication of excess power, 


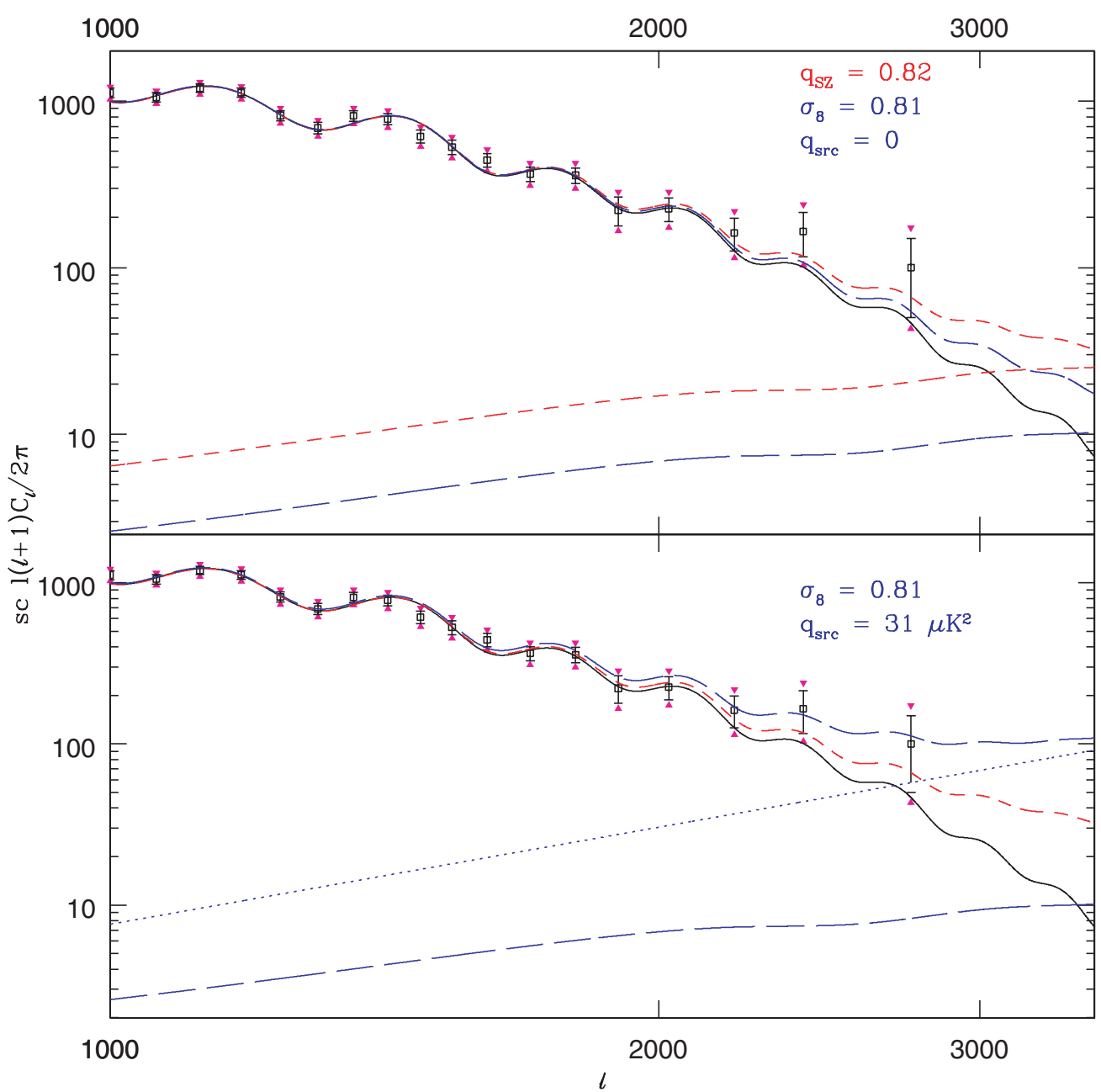

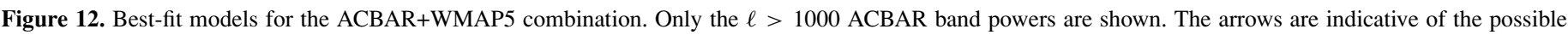

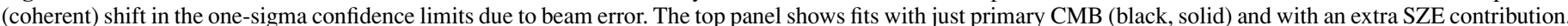

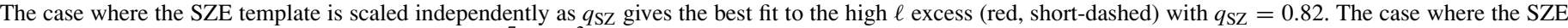

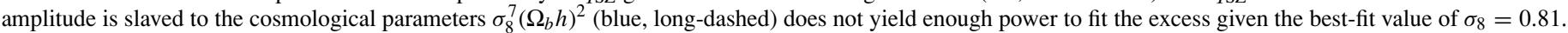

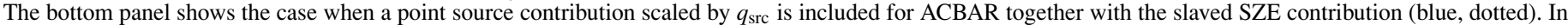
this case, the best-fit model has $\sigma_{8}=0.81$ determining the subdominant SZE contribution, and $q_{\mathrm{src}}=31 \mu \mathrm{K}^{2}$ determining the point source contribution.

we find a freely floating SZE amplitude results in $q_{\mathrm{SZ}}=$ $0.94_{-0.93}^{+0.35}$, with no effective lower bound. We can use the above relation of $q_{\mathrm{SZ}}\left(\sigma_{8}, \Omega_{b} h\right)$ to estimate a corresponding $\sigma_{8}^{(\mathrm{SZ})}=$ $0.97_{-0.13(-0.31)}^{+0.09(+0.13)}$, where in parentheses we have indicated the onesigma errors. This result is higher than, but compatible within the uncertainties, values obtained from the primary $\mathrm{CMB}$ fits alone: the ACBAR+WMAP5 fits in Table 4 give $\sigma_{8}=0.80_{-0.03}^{+0.03}$. The confidence limits of the derived $\sigma_{8}^{(\mathrm{SZ})}$ depend strongly on the choice of measure, which is here taken to be uniform in the amplitude $q_{\mathrm{SZ}}$. (This is evident in the translation of the relative flatness of the likelihood at low $q_{\mathrm{SZ}}$ to a "one-sigma detection" in $\sigma_{8}^{(\mathrm{SZ})}$.) When the SZE contribution is slaved to the $\sigma_{8}$ and $\Omega_{b} h$ values from the primary spectrum, there is no effect on $\sigma_{8}$. The high $\ell$ excess power is not significant enough to change the well-constrained value of $\sigma_{8}$ to the weakly preferred higher value. The effect of slaved and unslaved fits can be seen in Figure 12.

When the high $\ell$ band powers of CBI and BIMA are included in the analysis, there is a significant detection of excess power. Both the CBI and BIMA band powers are from $30 \mathrm{GHz}$ interferometric observations and have higher $f_{v}$ values than ACBAR. For the slaved case, the $\sigma_{8}$ value and errors are unchanged. For the floating case, we find $q_{\mathrm{SZ}}=0.69_{-0.11}^{+0.11}$, which maps to $\sigma_{8}^{(\mathrm{SZ})}=0.93_{-0.05}^{+0.04}$.

These central values and uncertainties are computed by transforming integrals of the likelihood $L\left(q_{\mathrm{SZ}}, \Omega_{b} h, \ldots\right)$ over the prior measures to confidence limits for $\sigma_{8}^{(\mathrm{SZ})}$. Exactly what measure to place on $q_{\mathrm{SZ}}$ and therefore on $\sigma_{8}^{(\mathrm{SZ})}$ is debatable. A measure uniform in $\alpha_{\mathrm{SZ}}=q_{\mathrm{SZ}}^{1 / 2}$, as we used in $\mathrm{K} 07$ and (Goldstein et al. 2003) translates into a measure $\propto q_{\mathrm{SZ}}^{-1 / 2} d q_{\mathrm{SZ}}$ that favors lower values of $\sigma_{8}^{(\mathrm{SZ})}$ than the measure uniform in $q_{\text {Sz }}$ that we have adopted.

As the cosmological parameters vary, the SZE template may depend on $\sigma_{8}$ and $\Omega_{b} h$, and certainly depends on the spectral index $n_{s}$ and astrophysical issues such as the history of energy injection into the cluster system. In a more complete treatment than that presented here, the shape should be modified along with the base cosmological parameters in the MCMC runs.

We caution that the derived $\sigma_{8}^{(\mathrm{SZ})}$ depends on the SZE template shape, its extension into the higher $\ell$ regime probed by BIMA, 


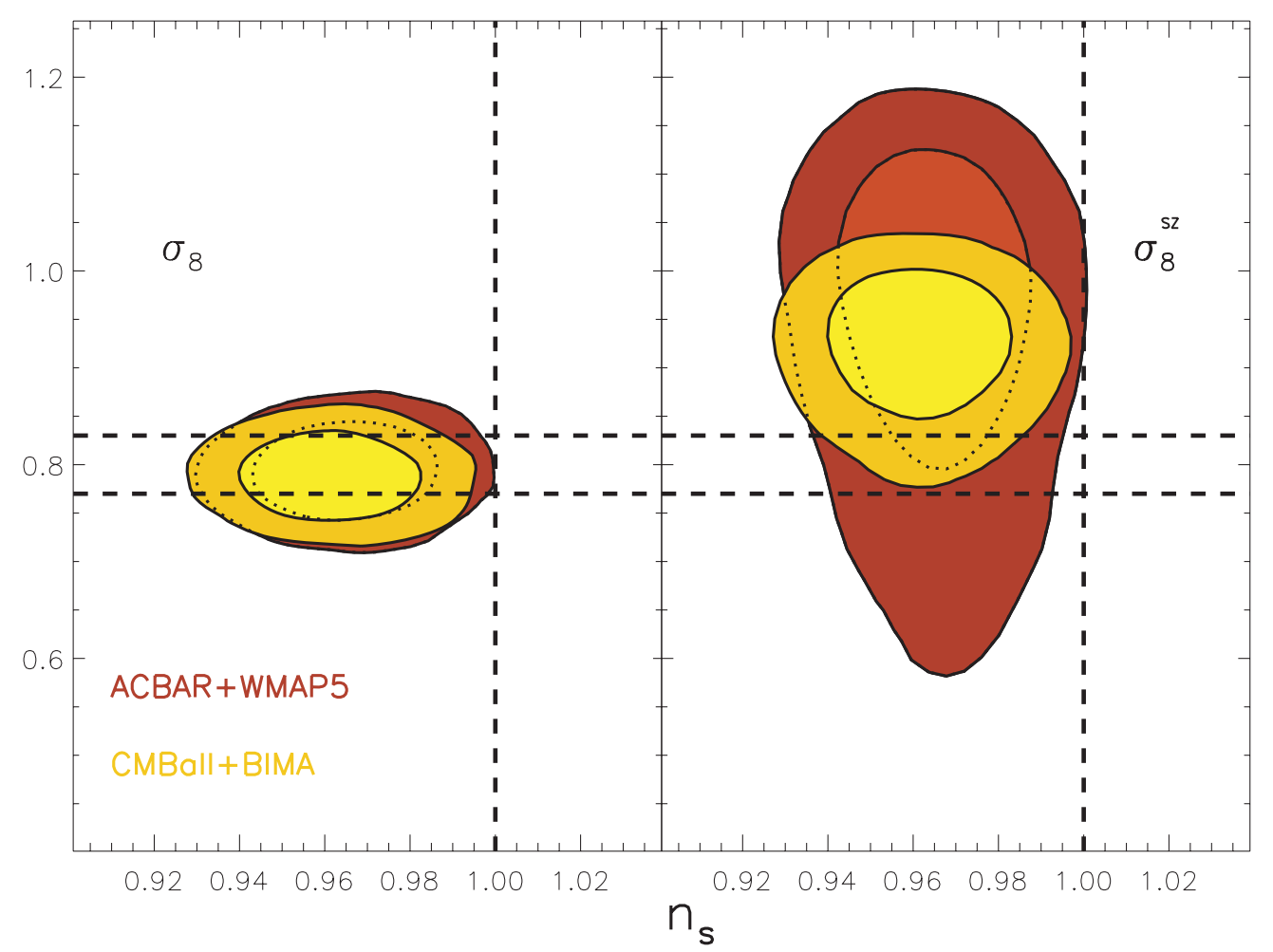

Figure 13. This figure contrasts the one and two-sigma contour intervals for $\sigma_{8}$ determined from the primary anisotropy component of the CMB (left) with the value inferred from the SZE template transformation of $q_{\mathrm{SZ}}$ into $\sigma_{8}^{(\mathrm{SZ})}$ (right), assuming a uniform prior measure in $q_{\mathrm{SZ}}$. Allowing for a point source contribution would decrease the tension between $\sigma_{8}$ and $\sigma_{8}^{\mathrm{SZ}}$ for the ACBAR+WMAP5 case. These panels also demonstrate the strength of the deviation of $n_{s}$ from unity for the flat $\Lambda \mathrm{CDM}$ model.

and the prior measure placed upon $q_{\mathrm{SZ}}{ }^{18}$ The modest decrease in $\log$ likelihood for the fit to the model when the SZE is taken into account is $\Delta \ln L=0.26$ for the ACBAR+WMAP5 combination and increases to $\Delta \ln L=1.91$ for CMBall+BIMA.

Regardless of the assumed prior, the interpretation of excess power as being due to the SZE results in a nonzero $\sigma_{8}^{(\mathrm{SZ})}$ for the CMBall+BIMA combination. Uncertainties for $\sigma_{8}^{(\mathrm{SZ})}$ are about a factor of two larger than for the $\sigma_{8}$ determined from the primary CMB data, and there is a tension at about the one-sigma level between the two median values. A visual summary of the results is shown in Figure 13 where we plot both $\sigma_{8}$ and $\sigma_{8}^{(\mathrm{SZ})}$ against the spectral index for a number of data combinations. The addition of LSS data does not significantly change these results. When the ACBAR dusty point source or SZE template marginalization is included, $\sigma_{8}$ decreases slightly for the CMBall data set.

Recent weak lensing results are in basic agreement with the primary $\sigma_{8}$ values when $\Omega_{m}=0.26 \pm 0.03$ from Table 4 is used. With $100 \mathrm{deg}^{2}$ of lensing data from the combination of the CFHT weak lensing legacy, RCS, Virmos-Descart, and GaBaDos surveys, Benjamin et al. (2007) get $\sigma_{8}\left(\Omega_{m} / 0.26\right)^{0.59}=0.80 \pm$ 0.05 . From the CFHT weak lensing legacy survey alone, $\mathrm{Fu}$ et al. (2007) get $\sigma_{8}\left(\Omega_{m} / 0.26\right)^{0.64}=0.753 \pm 0.043$, and get $\sigma_{8}\left(\Omega_{m} / 0.26\right)^{0.53}=0.82 \pm 0.084$ if only large-scale linearregime results are used. These weak lensing numbers are lower than past published results because of improved treatments of the redshift distribution of the lensed sources.

\footnotetext{
18 We also note that the non-Gaussian nature of the SZE signal was included in the BIMA results, but not in the CBI results. The non-Gaussian effect increases the sample variance and tends to open up the allowed range toward lower $\sigma_{8}$ values (Goldstein et al. 2003; Readhead et al. 2004a).
}

As shown in Figure 12, the marginal excess power in ACBAR is consistent with the combination of a point source contribution at the upper limit of the $150 \mathrm{GHz}$ extrapolations and an SZE template at the level predicted from the primary anisotropy $\sigma_{8}$ value. In this scenario, the cosmological parameters are virtually unchanged, but no explanation is provided for the CBI excess. The SZE analyses with a free-floating amplitude have not included additional foreground sources for CBI, BIMA, or ACBAR. The effect of radio sources extrapolated to $30 \mathrm{GHz}$ was included in the original CBI and BIMA results, and is unlikely to be an important contaminant for ACBAR. Dusty protogalaxies should not affect CBI and BIMA, but will contribute to the high $\ell$ ACBAR band powers. Given the uncertainty in the source contamination for ACBAR, the weak detection of excess power does not significantly support the SZE interpretation of the CBI+BIMA excess.

\subsection{Running Spectral Index}

There has been interest in the running of the spectral index $d n_{s} / d \ln k$ since the first release of WMAP data, which showed evidence for a significant negative running when combined with LSS and Lyman alpha forest observations (Spergel et al. 2003). With the precision of the new ACBAR data, we might expect improved constraints on running of the spectral index. To the basic six parameters in the minimal model, we add running of the spectral index $d n_{s} / d \ln k\left(k_{\star}\right)$ around the pivot point $k_{\star}=$ $0.05 \mathrm{Mpc}^{-1}$. We adopt the conventionally used uniform prior in $d n_{s} / d \ln k\left(k_{\star}\right)$, although in usual slow-roll-inflation models, the spectral index fluctuation $\delta n_{s} \propto \ln \left(k / k_{\star}\right) d n_{s} / d \ln k\left(k_{\star}\right)$ is typically restricted by $\left|1-n_{s}\right|$. Table 5 summarizes the parameter values when running is allowed and demonstrates that its inclusion has only a small effect on the other parameters. 
For WMAP5 only, we find $d n_{s} / d \ln k\left(k_{\star}\right)=-0.031_{-0.028}^{+0.029}$. The main tendency for the negative value comes from the low $\ell$ WMAP5 data. When the ACBAR data is added, the median value is similar, $d n_{s} / d \ln k\left(k_{\star}\right)=-0.037_{-0.022}^{+0.023}$, with a reduced error. The results are nearly identical for CMBall, but more compatible with no-running when LSS is added, $-0.016_{-0.018}^{+0.019}$. The precise measurement of the high $\ell$ CMB power spectrum provided by ACBAR is a potentially powerful constraint on running, but may also include significant contributions from secondary anisotropies and foregrounds. Therefore, in the last two columns, we have included the effect of marginalizing over the SZE or point source templates. In both cases, the mean value of the running becomes more negative and more significant at the one-sigma level. A visual representation of the impact of adding the ACBAR data is given in Figure 11, which shows the correlation between $n_{s}$ and $d n_{s} / d \ln k$. The scalar spectral index, $n_{s}\left(k_{\star}\right)=0.918 \pm 0.032$ for the WMAP5 + ACBAR combination, depends on the choice of pivot point $k_{\star}$; a smaller value would yield a higher result, while a higher one would give an even lower result.

\subsection{Tensor Modes}

We have run a limited number of cases including tensor modes, characterizing their strength relative to scalar perturbations by $r$ and fixing the tensor tilt by $n_{t} \approx-r / 8(1-r / 16)$. The most stringent upper limit for $r$ is given by the CMBall combination which yields $r<0.40$ (95\% confidence). This assumes a uniform prior measure for $r$, as is conventional in parameter estimation, although without much justification except that it is conservative. Adding LSS tightens this limit. Our result can be compared with those obtained by Dunkley et al. (2008), $r<0.43$ (95\% confidence) for WMAP5 alone.

\section{CONCLUSIONS}

We have used the complete ACBAR $150 \mathrm{GHz}$ data set to measure the $\mathrm{CMB}$ temperature anisotropy angular power spectrum. Over three seasons of observation, ACBAR dedicated $85 \mathrm{~K}$ detector hours to $\mathrm{CMB}$ observations at $150 \mathrm{GHz}$ and covered $1.7 \%$ of the sky. The data are calibrated by comparing CMB temperature maps for the largest ACBAR fields with those produced by WMAP5. This calibration is found to be consistent with the previous planet-based and RCW38-based calibrations, but with the temperature uncertainty reduced to $2.05 \%$. In the original preprint of this paper, the ACBAR band powers were calibrated through comparison with WMAP3. The new WMAP5 results became available during the review process, and we have updated the ACBAR band powers and cosmological parameters to take advantage of this new information. The ACBAR band powers are otherwise unchanged. The new WMAP5 parameters and calibration subtly changed the best fit WMAP5 and ACBAR model resulting in a decrease of the significance of the high $\ell$ excess in the ACBAR data.

The ACBAR band powers reported in Table 3 are the most sensitive measurements to date of CMB temperature anisotropies for multipoles between $\ell \sim 900$ and 3000. In this data, the fourth and fifth acoustic peaks are significantly detected for the first time. These precise measurements of the CMB temperature anisotropies at high $\ell$ are consistent with a spatially flat, dark energy-dominated $\Lambda \mathrm{CDM}$ cosmology. Including the effects of $\mathrm{CMB}$ weak lensing in the computation of model power spectra improves the fits to the combined ACBAR+WMAP5 data. The excellent fit of the $\Lambda \mathrm{CDM}$ cosmological model to the combined ACBAR+WMAP5 data at $\ell \lesssim 2000$ is a strong confirmation of the standard cosmological paradigm and gives us confidence in the resulting parameter values. The ACBAR data favor higher median values of $\sigma_{8}, \Omega_{m}$, and $\theta$ than those preferred by WMAP3; however, with the improved WMAP5 results for $\ell>650$, this tension has been resolved. The parameter values remain stable with the inclusion of additional CMB and LSS data sets, with or without the marginalization over an SZE or point source template for ACBAR. For example, $\sigma_{8} \sim 0.80$ holds for all parameter variations in Table 4 , and even in Table 5 with the inclusion of a running spectral index.

We have performed strict jackknife tests with the data, and find that the results are free of significant systematic errors. We have projected templates derived from the FDS99 dust model and the PMN radio source catalog from the ACBAR maps before estimating the band powers and find the residual contributions from these foregrounds to be negligible at the current sensitivity. The contribution of dusty protogalaxies is expected to be insignificant for all but the few highest $\ell$ band powers, but remains poorly constrained due to our incomplete knowledge of the spectral dependence of these sources.

Secondary anisotropies are expected to become important at small angular scales. The ACBAR band powers are slightly higher $(1.1 \sigma)$ than expected for the primary CMB anisotropy at multipoles above $\ell \sim 2000$. We expect that some of this power results from contamination by dusty protogalaxies. However, the combined signal is considerably smaller than the significant detection of excess power reported by the CBI experiment at $30 \mathrm{GHz}$. A joint analysis of the $\mathrm{CBI}$ and ACBAR band powers in the multipole range of $2000 \lesssim \ell \lesssim 3000$ argues strongly against the CBI excess having the spectrum of primary CMB anisotropy. These results are consistent with the excess power seen by CBI, being due to either the Sunyaev-Zel'dovich effect or radio source contamination. Higher sensitivity observations over a broad range of frequencies are necessary in order to fully characterize CMB secondary anisotropies and eliminate potential foreground contamination.

The ACBAR program has been primarily supported by NSF office of polar programs grants OPP-8920223 and OPP0091840. This research used resources of the National Energy Research Scientific Computing Center, which is supported by the Office of Science of the U.S. Department of Energy under Contract No. DE-AC03-76SF00098. Some computations were performed on the Canada Foundation for Innovation funded CITA Sunnyvale cluster. Chao-Lin Kuo acknowledges support from a NASA postdoctoral fellowship, and Marcus Runyan acknowledges support from a Fermi fellowship. Christian Reichardt acknowledges support from a National Science Foundation Graduate Research Fellowship. Some of the results in this paper have been derived using the HEALPix (Górski et al. 2005) package. We thank members of the BOOMERANG team, in particular Brendan Crill, Bill Jones, and Tom Montroy for providing access to the $\mathrm{B} 03$ data, the pipeline used to generate simulation maps, and assistance with its operation. We thank Antony Lewis for discussions about ways to parameterize tests for weak lensing in the data.

\section{APPENDIX A \\ CALIBRATION}

The calibration used in K07 was linked to the Boomerang03 (B03) calibration with observations of RCW38. In this section, 
we describe a new calibration using an $a_{\mathrm{lm}}$-based comparison of CMB structure observed by WMAP5 and ACBAR in 2005. This method is inspired by the calibration scheme used to calibrate B03 to WMAP. The 2005 calibration is carried to other years by an ACBAR-ACBAR power spectrum comparison on fields observed in both years. The WMAP-ACBAR crosscalibration method is described below, with a detailed account of uncertainty in Table 6.

Table 6

Error Budget for the $a_{\ell m}$-based ACBAR Calibration

\begin{tabular}{lc}
\hline \hline Source & Uncertainty (\%) \\
\hline Statistical error in the calibration ratio & 1.10 \\
$\ell$ dependence of the calibration ratio & 1.1 \\
Statistical error in the transfer function of the calibration ratio & 0.35 \\
Uncertainty in the WMAP $B_{\ell}$ & 0.5 \\
Relative pointing uncertainty & 1.0 \\
Uncertainty in the tear-to-year ACBAR calibration & 0.3 \\
Uncertainty in the transfer function for the power spectrum & 0.5 \\
Contamination from foregrounds & 0.2 \\
WMAP5's absolute calibration & 0.2 \\
Overall & 2.05 \\
\hline
\end{tabular}

Notes. Calibration of ACBAR using the WMAP5 temperature maps has multiple potential sources of error, tabulated here for reference. The dominant calibration uncertainties are due to noise in the WMAP maps at the angular scales used for calibration. The uncertainty in the ACBAR beam function is comparable to the calibration uncertainty.

\section{APPENDIX B}

WMAP-ACBAR

Calibrating with the CMB temperature anisotropies has two main advantages. The first is that the calibration of the WMAP temperature maps (at $0.2 \%$ in temperature) is an order of magnitude more precise than the flux calibration of the calibration sources ACBAR used in previous releases. The second advantage is that the anisotropies have the same spectrum as what is being calibrated, rendering the large frequency gap between WMAP and ACBAR irrelevant.

The two experiments have different scan patterns, noise, beam widths, and spatial filters that will affect the measured flux. In this analysis, we assume that the WMAP5 maps are effectively unfiltered except for the instrumental beam function. The two maps can be represented as

$$
\begin{gathered}
S_{i}^{\mathrm{WMAP}}=\int T(x) B_{\mathrm{WMAP}}\left(x_{i}-x\right) d x+N_{i}^{\mathrm{WMAP},} \\
S_{i}^{\mathrm{ACBAR}}=F_{i j} \int T(x) B_{\mathrm{ACBAR}}\left(x_{j}-x\right) d x+N_{i}^{\mathrm{ACBAR},}
\end{gathered}
$$

where $T$ is the underlying CMB signal, $N$ is the instrumental noise, $B$ is the beam function, and $F_{i j}$ is the ACBAR filter matrix as defined in Section 3. We reduce the filtering differences by resampling the WMAP map using the ACBAR pointing information and applying the ACBAR spatial filtering to generate an "ACBAR-filtered" WMAP map

$$
S_{i}^{\mathrm{WMAP}-\text { equivalent }}=F_{i j}\left(\int T(x) B_{\mathrm{WMAP}}\left(x_{j}-x\right) d x+N_{i}^{\mathrm{WMAP}}\right) .
$$

The results of applying this algorithm to the B03 map are shown in Figure 14. We choose to do the absolute calibration via cross-power spectra rather than a direct pixel-to-pixel comparison of the maps. Using cross-spectra significantly reduces the impact of the noise model on the result. The significant beam differences between the experiments are more naturally dealt with in multipole space than in pixel space. We construct the ratio from the filtered maps,

$$
R=\Re\left(\frac{a_{\ell m}^{\mathrm{WMAP}-X *} * a_{\ell m}^{\mathrm{ACBAR}-Z}}{a_{\ell m}^{\mathrm{ACBAR}-Y *} * a_{\ell m}^{\mathrm{ACBAR}-Z}\left(B_{\ell}^{\mathrm{WMAP}-X} / B_{\ell}^{\mathrm{ACBAR}}\right)}\right),
$$

where $X$ can denote either the V- or W-band map for WMAP and $Y / Z$ marks either of two noise-independent ACBAR combinations. There is a narrow $\ell$ range from $256-512$ useful for calibration. The range is limited at high $\ell$ by the rapidly falling WMAP beam function and at low $\ell$ by the ACBAR polynomial filtering, which acts as a high-pass filter. We choose to use the WMAP $\mathrm{V}$ and $\mathrm{W}$ bands to take advantage of their smaller beam size.

Monte Carlo simulations are used to determine the transfer function of this estimator. We generate CMB sky simulations convolved with the respective instrumental beam functions using the Healpix ${ }^{19}$ library. We resample each realization and apply the ACBAR filtering matrix described above to generate equivalent maps for each field. We expected and found a small intrinsic bias as the beam convolution and filtering operations do not commute: $B^{\mathrm{ACBAR}} * F_{i j} B^{\mathrm{WMAP}} \neq B^{\mathrm{WMAP}} * F_{i j} B^{\mathrm{ACBAR}}$. We correct the real data by the $\ell$-dependent transfer function measured in these simulations. The technique is easily adapted to estimate the error caused by pointing uncertainties and to confirm that the estimator is unbiased with the inclusion of noise. The derived error in the transfer function is listed in Table 6.

Foreground sources have the potential to systematically bias a calibration bridging 60-150 GHz. Radio sources, synchrotron emission, dust, and free-free emission all have a distinctly different spectral dependence than the CMB, which could lead to a calibration bias. This risk is ameliorated by the positioning of the ACBAR fields in regions of exceptionally low foregrounds. Bright radio sources detected in either experiment are masked and excluded from the calibration. The calibration proved insensitive to the exact threshold for source masking. We use the MEM foreground models in Hinshaw et al. (2006) to estimate the rms fluctuations of each foreground relative to the CMB fluctuations and find that the free-free and synchrotron fluctuations are less than $0.1 \%$ of the CMB fluctuations in all frequency bands, while dust emission can reach $1.5 \%$ of $\mathrm{CMB}$ fluctuations in the $150 \mathrm{GHz}$ maps. We test the effects of the most significant foreground, dust, by adding the FDS99 dust model (Finkbeiner et al. 1999) to a set of CMB realizations. The resultant maps are passed through a simulated pipeline as outlined in the previous paragraph. We find that the addition of dust does not introduce a detectable bias with an uncertainty of $0.2 \%$.

We perform a weighted average of the measured calibration ratio across all $\ell$ bin, field and band combinations after correcting for the estimated signal-only transfer functions. We estimate the calibration error to be $1.97 \%$ for the 2005 data. Table 6 tabulates the contributing factors and error budget. We then propagate this $a_{\ell \mathrm{m}}$-based calibration to the CMB observations done in 2001 and 2002.

\footnotetext{
19 http://healpix.jpl.nasa.gov.
} 

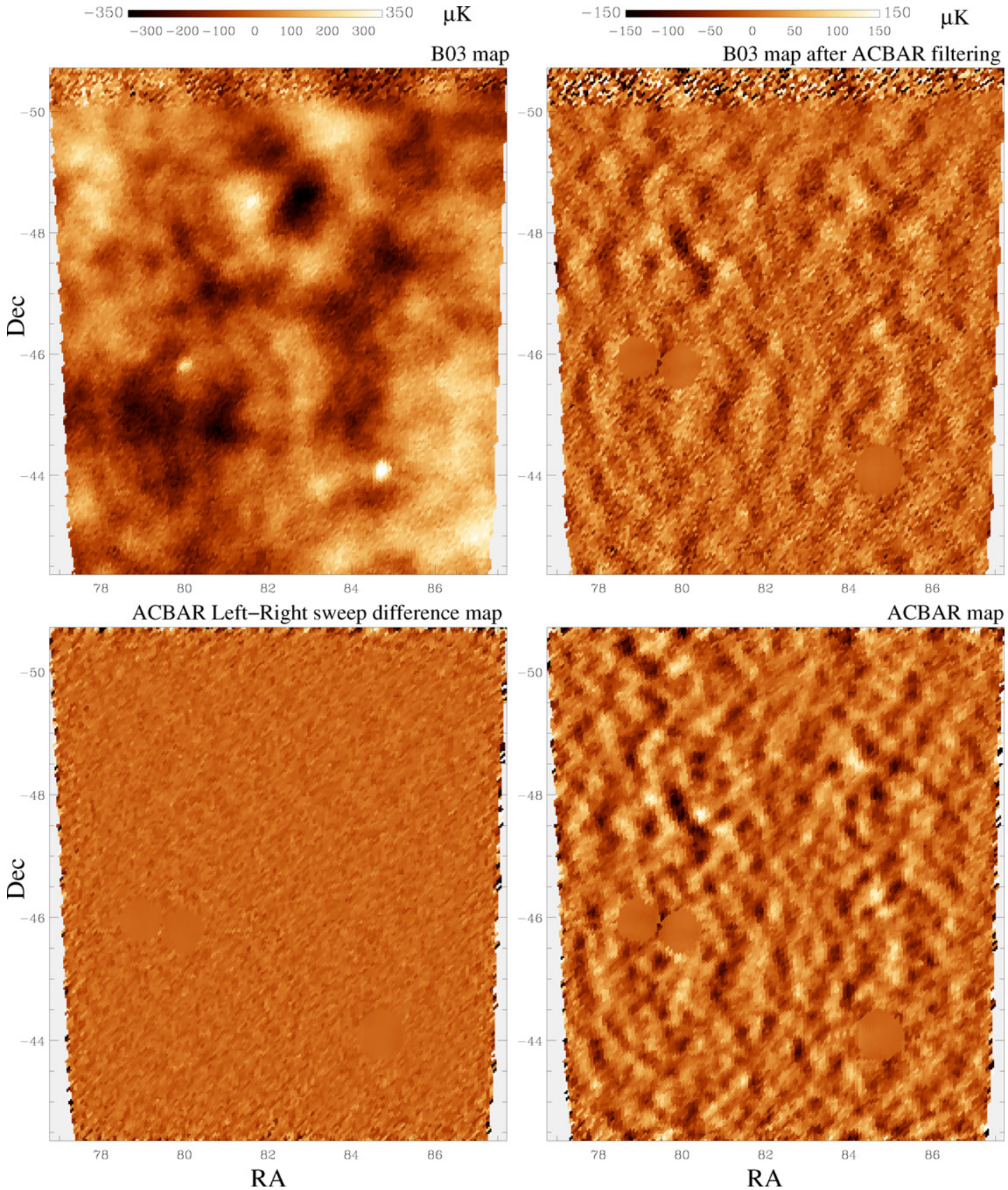

Figure 14. Comparison of observations of the CMB8 field made with B03 and ACBAR. This field lies in the deep region of the B03 map. The top two maps are from B03. The bottom two maps are from ACBAR. In the top left panel is the B03 map of the CMB8 field. The dynamic range of this map is greater than that of the other three figures. The increased noise at one edge marks the edge of the B03 deep coverage. The ACBAR filtering is applied to the B03 map to create the map in the top right panel. Directly below it in the bottom right panel is the ACBAR map of same region. Note the clear correspondence between the CMB anisotropies observed by B03 and ACBAR. Three bright point sources have been masked. An ACBAR left-right sweep difference map is shown in the bottom left panel. The power spectrum of this map (and the other nine fields) is plotted in Figure 3.

\section{APPENDIX C}

\section{ACBAR 2001-2002 AND 2002-2005 CROSS-CALIBRATIONS}

We propagate the 2005 calibration into 2001 and 2002 by comparing the 2001 observations of the CMB2 field to the overlapping 2002 CMB4 field, and the 2002 observations of the CMB5 field to the 2005 observations of the CMB5 field. A power spectrum is calculated for each overlapping region and the ratio of the band powers is used to derive a crosscalibration. The procedures used are outlined in more detail in K07. We use the same relative calibration for 2001 as K07: $T_{2001} / T_{2002}=1.238 \pm 0.067$. We find cross-calibration factor for 2002 to be $T_{2005} / T_{2002}=1.035 \pm 0.025$. We apply these corrections to the data and determine the overall calibration uncertainty to be $2.05 \%$ (in temperature units) based primarily on the uncertainties associated with WMAP/ACBAR-2005 cross-calibration.

\section{REFERENCES}

Ade, P., et al. 2007, ArXiv e-prints, 705

Albrecht, A., Coulson, D., Ferreira, P., \& Magueijo, J. 1996, Phys. Rev. Lett., 76,1413

Benjamin, J., et al. 2007, submitted (arXiv:astro-ph/0703570)

Bond, J. R., Jaffe, A. H., \& Knox, L. 1998, Phys. Rev. D, 57, 2117 
Bond, J. R., Jaffe, A. H., \& Knox, L. 2000, ApJ, 533, 19 (arXiv: astro-ph/9808264)

Bond, J. R., et al. 2005, ApJ, 626, 12

Borys, C., Chapman, S., Halpern, M., \& Scott, D. 2003, MNRAS, 344, 385

Bridle, S. L., Lewis, A. M., Weller, J., \& Efstathiou, G. 2003, MNRAS, 342, L72

Burles, S., Nollett, K. M., \& Turner, M. S. 2001, Phys. Rev. D, 63, 063512 (astro-ph/0008495)

Calabrese, E., Slosar, A., Melchorri, A., Smoot, G. F., \& Zahn, O. 2008, 803, ArXiv e-prints

Cole, S., et al. 2005, MNRAS, 362, 505

Cooray, A., Hu, W., \& Tegmark, M. 2000, ApJ, 540, 1

Cooray, A., \& Melchiorri, A. 2002, Phys. Rev. D, 66, 083001

Coppin, K., et al. 2006, MNRAS, 372, 1621

Dawson, K. S., Holzapfel, W. L., Carlstrom, J. E., Joy, M., \& LaRoque, S. J. 2006, ApJ, 647, 13

Dickinson, C., et al. 2004, MNRAS, 353, 732

Dunkley, J., et al. 2008, ArXiv e-prints, 803

Finkbeiner, D. P., Davis, M., \& Schlegel, D. J. 1999, ApJ, 524, 867

Fu, L., et al. 2007, submitted (arXiv:0712.0884)

Goldstein, J. H., et al. 2003, ApJ, 599, 773

Górski, K. M., Hivon, E., Banday, A. J., Wandelt, B. D., Hansen, F. K., Reinecke, M., \& Bartelmann, M. 2005, ApJ, 622, 759

Greve, T. R., Ivison, R. J., Bertoldi, F., Stevens, J. A., Dunlop, J. S., Lutz, D., \& Carilli, C. L. 2004, MNRAS, 354, 779

Griffiths, L. M., Kunz, M., \& Silk, J. 2003, MNRAS, 339, 680

Halverson, N. W., et al. 2002, ApJ, 568, 38

Hanany, S., et al. 2000, ApJ, 545, L5

Hinshaw, G., et al. 2006, ApJ, submitted (arXiv:astro-ph/0603451)

Hinshaw, G., et al. 2008, ArXiv e-prints, 803

Hirata, C. M., Ho, S., Padmanabhan, N., Seljak, U., \& Bahcall, N. 2008, ArXiv e-prints, 801

Huffenberger, K. M., Eriksen, H. K., \& Hansen, F. K. 2006, ArXiv Astrophysics e-prints

Jones, W. C., et al. 2006, ApJ, 647, 823

Knox, L. 1999, Phys. Rev. D, 60, 103516 (arXiv:astro-ph/9902046)
Knox, L., Holder, G., \& Church, S. 2004, ApJ, 612, 96

Komatsu, E., \& Seljak, U. 2002, MNRAS, 336, 1256

Kuo, C. L., et al. 2007, ApJ, 664, 687

Kuo, C. L., et al. 2004, ApJ, 600, 32

Laurent, G. T., et al. 2005, ApJ, 623, 742

Leach, S. M., \& Liddle, A. R. 2003, MNRAS, 341, 1151

Leitch, E. M., Kovac, J. M., Halverson, N. W., Carlstrom, J. E., Pryke, C., \& Smith, M. W. E. 2005, ApJ, 624, 10

Lewis, A., \& Bridle, S. 2002, Phys. Rev. D, 66, 103511

Lewis, A., Challinor, A., \& Lasenby, A. 2000, ApJ, 538, 473

Maloney, P. R., et al. 2005, ApJ, 635, 1044

Mason, B. S., et al. 2003, ApJ, 591, 540

Montroy, T. E., et al. 2006, ApJ, 647, 813

Mukherjee, P., \& Wang, Y. 2003, ApJ, 599, 1

Nolta, M. R. 2008, ArXiv e-prints, 803

Peiris, H. V., et al. 2003, ApJS, 148, 213

Piacentini, F., et al. 2006, ApJ, 647, 833

Readhead, A. C. S., et al. 2004a, ApJ, 609, 498

Readhead, A. C. S., et al. 2004b, Science, 306, 836

Riess, A. G., et al. 2004, ApJ, 607, 665

Riess, A. G., et al. 2007, ApJ, 659, 98

Runyan, M. C., et al. 2003, ApJS, 149, 265

Schlegel, D. J., Finkbeiner, D. P., \& Davis, M. 1998, ApJ, 500, 525

Scott, D., \& White, M. 1999, A\&A, 346, 1

Seljak, U. 1996, ApJ, 463, 1

Sievers, J. L., et al. 2005, ArXiv Astrophysics e-prints

Smith, K. M., Zahn, O., \& Doré, O. 2007, Phys. Rev. D, 76, 043510

Spergel, D. N., et al. 2006, ArXiv Astrophysics e-prints

Spergel, D. N., et al. 2003, ApJS, 148, 175

Subramanian, K., Seshadri, T. R., \& Barrow, J. D. 2003, MNRAS, 344, L31

Tegmark, M. 1997, Phys. Rev. D, 55, 5895

Tegmark, M., et al. 2006, Phys. Rev. D, 74, 123507

White, M., Hernquist, L., \& Springel, V. 2002, ApJ, 579, 16

White, M., \& Majumdar, S. 2004, ApJ, 602, 565

Wright, A. E., Griffith, M. R., Burke, B. F., \& Ekers, R. D. 1994, ApJS, 91, 111

Zhang, P., Pen, U., \& Wang, B. 2002, ApJ, 577, 555 